\title{
Cirbp-PSD95 Axis Protects Against Hypobaric Hypoxia-Induced Aberrant Morphology of Hippocampal Dendritic Spines and Cognitive Deficits
}

\section{Yang Zhou}

Fourth Military Medical University: Air Force Medical University

Huanyu Lu

Fourth Military Medical University: Air Force Medical University

Ying Liu

Fourth Military Medical University: Air Force Medical University

\section{Zaihua Zhao}

Fourth Military Medical University: Air Force Medical University

Qian Zhang

Fourth Military Medical University: Air Force Medical University

Chong Xue

Fourth Military Medical University: Air Force Medical University

\section{Yuankang Zou}

Fourth Military Medical University: Air Force Medical University

\section{Zipeng Cao}

Fourth Military Medical University: Air Force Medical University

Wenjing Luo ( $\triangle$ luowenj@fmmu.edu.cn )

Fourth Military Medical University: Air Force Medical University

https://orcid.org/0000-0003-4826-3961

\section{Research}

Keywords: Hypoxia, Cognitive impairment, Dendritic spine morphology, cold-inducible RNA - binding protein, PSD95

Posted Date: November 25th, 2020

DOl: https://doi.org/10.21203/rs.3.rs-113452/v1

License: (9) This work is licensed under a Creative Commons Attribution 4.0 International License. Read Full License 
Version of Record: A version of this preprint was published at Molecular Brain on August 21st, 2021. See the published version at https://doi.org/10.1186/s13041-021-00827-1. 
1 Cirbp-PSD95 axis protects against hypobaric hypoxia-induced aberrant

3 Yang Zhou ${ }^{1, \#}$, Huanyu $\mathrm{Lu}^{2, \#}$, Ying $\mathrm{Liu}^{3}$, Zaihua Zhao ${ }^{4}$, Qian Zhang ${ }^{5}$, Chong Xue ${ }^{6}$,

$4 \quad$ Yuankang Zou ${ }^{7}$, Zipeng $\mathrm{Cao}^{8, *}$, Wenjing $\mathrm{Luo}^{9, *}$

1 Department of Occupational and Environmental Health and the Ministry of Education Key Lab of Hazard Assessment and Control in Special Operational Environment, School of Public Health, Fourth Military Medical University, China. Email: zhouyang@fmmu.edu.cn.

2 Department of Occupational and Environmental Health and the Ministry of Education Key Lab of Hazard Assessment and Control in Special Operational Environment, School of Public Health, Fourth Military Medical University, China. Email: Lu_Huanyu@163.com.

3 Department of Occupational and Environmental Health and the Ministry of Education Key Lab of Hazard Assessment and Control in Special Operational Environment, School of Public Health, Fourth Military Medical University, China. Email: imLYing2015@163.com.

4 Department of Occupational and Environmental Health and the Ministry of Education Key Lab of Hazard Assessment and Control in Special Operational Environment, School of Public Health, Fourth Military Medical University, China. Email: 595055591@qq.com.

5 Department of Occupational and Environmental Health and the Ministry of Education Key Lab of Hazard Assessment and Control in Special Operational Environment, School of Public Health, Fourth Military Medical University, China. Email: zq0322@qq.com.

6 Department of Occupational and Environmental Health and the Ministry of Education Key Lab of Hazard Assessment and Control in Special Operational Environment, School of Public Health, Fourth Military Medical University, China. Email: xuechong456@fmmu.edu.cn.

7 Department of Occupational and Environmental Health and the Ministry of 


\section{5}

Education Key Lab of Hazard Assessment and Control in Special Operational Environment, School of Public Health, Fourth Military Medical University, China. Email: sydzouyk@163.com.

8 Department of Occupational and Environmental Health and the Ministry of Education Key Lab of Hazard Assessment and Control in Special Operational Environment, School of Public Health, Fourth Military Medical University, China. Email: czpeng@fmmu.edu.cn.

9 Department of Occupational and Environmental Health and the Ministry of Education Key Lab of Hazard Assessment and Control in Special Operational Environment, School of Public Health, Fourth Military Medical University, China. Email: luowenj@fmmu.edu.cn.

\# Yang Zhou and Huanyu Lu contributed equally to this work.

Short title: Cirbp-PSD95 alleviates hypoxia induced dendritic spines abnormality

* Corresponding author at:

Zipeng Cao, Department of Occupational and Environmental Health, School of Public Health, Fourth Military Medical University, 169 Changlexi Road, Xi'an 710032, China. Phone: 86-29-84711325. E-mail: czpeng@fmmu.edu.cn. ORCID: 0000-0002-08782815.

Wenjing Luo, Department of Occupational and Environmental Health, School of Public Health, Fourth Military Medical University, 169 Changlexi Road, Xi'an 710032, China. Phone: 86-29-84774863, E-mail: luowenj@fmmu.edu.cn. ORCID: 0000-0003-48263961. 


\section{Abstract}

Hypobaric hypoxia $(\mathrm{HH})$ is a typical characteristic of high altitude environment and causes a spectrum of pathophysiological effects, including headaches, gliovascular dysfunction and cognitive slowing. Here, we sought to understand the mechanisms underlying cognitive deficits under $\mathrm{HH}$ exposure. Our results showed that $\mathrm{HH}$ exposure impaired cognitive function and suppressed dendritic spine density accompanied with increased neck length in both basal and apical hippocampal CA1 region neurons. The expression of PSD95, a critical synaptic scaffolding molecule, is down-regulated by hypoxia exposure and post-transcriptionally controlled by cold-inducible RNA-binding protein (Cirbp) through 3'-UTR region binding. PSD95 expressing alleviates hypoxiainduced neuron dendritic spine plasticity abnormality and memory impairment. Moreover, overexpressed Cirbp in hippocampus rescues hypoxia-induced loss of PSD95 and attenuates hypoxia-induced dendritic spine injury and cognitive outcomes. Thus, our findings reveal a novel mechanism where Cirbp-PSD-95 axis appears to play a key role in hypoxia-induced cognitive abilities impairment in brain.

Keywords Hypoxia; Cognitive impairment; Dendritic spine morphology; Coldinducible RNA-binding protein; PSD95. 


\section{Introduction}

Millions of people permanently reside in high altitude (HA) environment, which cover about one-fifth of the earth's surface, and many have been there for generations (1). In recent years, migration to HA regions has been an increasingly common activity, including travelers, mountaineers, military personnel, miners and skiers. Exposure to hypobaric hypoxia encountered during ascent to high altitude is commonly associated with neurophysiological disturbances including insomnia, dizziness, nausea, and cognitive impairment, as it is associated with a decreased partial pressure of oxygen leading to reduced oxygen delivery to brain. $(2,3)$. Researchers have reported that humans may suffer from neuropsychological impairment at HA (4). We have shown that executive function characterized by working memory and psychomotor function is impaired upon HA exposure (5). However, the underlying mechanisms of hypoxia on cognitive deficits remain poorly understood.

Dendritic spines are a morphological feature of the majority of excitatory synapses in the mammalian neocortex, and dendritic spine remodeling is thought to underlie synaptic plasticity. Abnormal dendritic spine plasticity may contribute to neurological disorders. Whether and how long-time physiological hypoxia leads to dendritic spine deficits has yet to be determine.

The protein, PSD95, is an integral part of the post-synaptic density and provides the scaffold for clustering and stabilizing glutamate receptors. PSD95 expression is increasing to maximum levels in the adult, when spine stability and synaptic maturity are at their peak, and PSD95 overexpression leads to an increase in spine density as well as average spine size, and alleviates brain cognitive deficits. Thus, here we set to determine whether PSD95 is a potential therapeutic target for mitigating hypoxiainduced learning-memory impairment.

Cold-inducible RNA-binding protein (Cirbp) has been initially identified as a member of cold shock proteins and expresses in a variety of mammalian cells, participating in various biological functions (6). Endogenous and environmental stressors, including hypoxia have been shown to regulate the expression of Cirbp (7). Cirbp is an RNA binding protein and has been reported to modulate gene expression 
posttranscriptionally (8). Our previous published work has shown that Cirbp sustained the proliferation of neural stem cells under hypoxic exposure, and the level of Cirbp was repressed under hypoxia condition (9). However, it remains uncertain whether Cirbp can regulate the dendritic spine morphology, affecting hypoxia-induced cognitive dysfunction. Therefore, we investigated the role of Cirbp-PSD95 axis in hypoxiainduced dendritic spines plasticity abnormality and learning-memory impairment.

\section{Results}

Hypoxia impaired cognitive function and dendritic spines morphology

To assess the effect of $\mathrm{HH}$ on cognitive function, we performed Morris water maze (MWM), step-down inhibitory avoidance test (SIAT) and novel object recognition test (NORT) (Figure 1A). Mice were subjected to the MWM analysis to detect the effect of hypoxic exposure on spatial reference memory. In the hidden platform trail, animals exposed to hypoxia showed increased escape latency, as shown in Figure 1B and C. The SIAT analysis was employed to evaluate the working memory ability (Figure 1D). After 14 days, chronic hypoxia exposed mice had shorter step-down time compared to mice in normoxic group. In NORT analysis, which tests episodic memory capacity, there were no difference in the total distance and the mean speed of mice in two groups (Figure 1E, Supplementary Figure 1A and B). However, the exploration time on novel objects in the control group was significantly longer than in the hypoxic group (Figure 1F), and the discrimination index was weakened in the hypoxic group (Figure 1G). All above, these findings provided that hypoxic exposure leads to aggravated cognitive impairment under this condition.

Dendritic spines represent primary postsynaptic sites of excitatory synaptic inputs to pyramidal neurons, and studying densities and morphology of these structures provides invaluable information on morphofunctional characteristics of cognitive ability $(10,11)$. Next, the morphology of dendritic spines remodeling was examined, showing there was no difference in the intersection number of the dendritic arborizations between two groups (Figure 1H and I). Next, we analyzed basal and apical spines separately, and found that the hypoxia significantly suppressed dendritic spine 
density (Figure 1J, K) and increased neck length in both basal and apical hippocampal CA1 region neurons (Figure 1L). Taken together, chronic hypoxic exposure impaired both murine memory ability and hippocampal dendritic spine's morphology.

PSD95 alleviates hypobaric hypoxia-induced aberrant morphology of dendritic spines and cognitive deficits

To explore putative mechanism of the effect of hypoxic exposure on cognition and dendritic spines, expression of PSD95 was evaluated by western blot. Western blot analysis established decreased PSD95 protein levels in hippocampus under hypoxic condition (Figure 2A). RT-PCR analysis revealed that the mRNA level of PSD95 was also down-regulated (Supplementary Figure 1C). To determine whether the observed decrease in PSD95 protein expression was associated with the hypoxia-induced memory deficits and dendritic spine abnormalities, we injected AAV-PSD95-GFP into mouse hippocampal CA1 region (Figure $2 \mathrm{~B}$ ). The protein was successfully expressed in the CA1 region (Figure 2C), and exogenous PSD95 protein was expressed in the hippocampus (Figure 2D). MWM analysis revealed that overexpressed PSD95 markedly shortened the escape latency, and ameliorated hypoxia-induced memory impairment (Figure 2E, F). Furthermore, increased PSD95 protein levels significantly prolonged the platform leaving time after hypoxia exposure in SIAT experiment (Figure 2G). Furtherly, we corroborated that over-expression of PSD95 reversed the decreased density of apical spines in the dendritic spines of the CA1 hippocampal region (Figure $2 \mathrm{H}$, I), confirming the protective role of PSD95 on hypobaric hypoxia-induced dendritic spine morphology injury.

PSD95 is post-transcriptionally regulated by Cirbp under hypoxic condition

To elucidate the mechanism underlying the dendritic spine abnormalities caused by exposure to hypobaric hypoxia, we evaluated spine morphology in primary hippocampal neurons firstly. Primary cultured neurons were transfected with fluorescent-GFP and cultured in the normal $\left(21 \% \mathrm{O}_{2}\right)$ and hypoxia $\left(1 \% \mathrm{O}_{2}\right)$ conditions for $24 \mathrm{~h}$. There were no cell death or swelling in morphological observation (Supplementary Figure 1D). Consistent with our experiments in vivo, we found that hypoxic exposure resulted in reduction in spine density in cultured neurons (Figure 3A), 
and the level of PSD95 was concomitantly and significantly decreased in the hypoxic group when compared with the control group (Figure 3B). However, under hypoxic conditions, inconsistent with the noted down-regulation of protein level, PSD95 expression at the RNA level was elevated in primary hippocampal neurons and HT-22 cell lines (Figure 3C and Supplementary Figure 1E, F). These results indicated that under hypoxic conditions, the expression of PSD95 may be determined by its translation or its protein stability regulation, which may lead to the compensatory increase of PSD95 mRNA. Next, we applied Cycloheximide (CHX) treatments (5 $\mu \mathrm{g} / \mathrm{ml}, 0,8,16 \mathrm{~h}$ ) under hypoxic exposure to determine the PSD95 protein degradation rate. Western blot affirmed that PSD95 protein was dramatically down-regulated upon CHX treatment, and there were no statistically significant differences in its protein levels upon normoxic or hypoxic conditions (Figure 3D). Thus, we surmised whether hypoxia suppressed PSD95 expression on translational level.

As RNA-binding protein (RBP) plays a core role in post-transcriptional regulation, next, we determined the timing and magnitude of protein expression (12). We analyzed RBP binding motif distribution in PSD95 mRNA, noting several Cirbp binding sites in the 3'UTR region of PSD95 mRNA (Figure 3E). To further verify that Cirbp regulates the PSD95 expression through binding to its motif in 3'UTR region, we divided the PSD95 3'UTR into three fragments and constructed a series of luciferase reporter vectors (3'UTR A, B and C fragments). Cirbp motif sites were only present in B, and $\mathrm{C}$ fragments, and the luciferase assay showed that Cirbp significantly increased the luciferase activity of fragment $\mathrm{B}$ and $\mathrm{C}$, but did not affect the activity of fragment $\mathrm{A}$ (Figure 3F). Consistent with hypoxia-induced decrease in PSD95 protein expression, the protein of murine hippocampal Cirbp was significantly down-regulated under hypoxic conditions (Supplementary Figure 2A). Similar findings were noted both in primary cultured hippocampal neurons (Figure 3G) and HT-22 cells (Supplementary Figure 2B). Next, we performed RNA immunoprecipitation (RIP) to isolate mRNA bound to endogenous Cirbp in HT-22 cells and utilized specific primers to identify the Cirbp motif regions by RT-PCR analysis. The data demonstrated that the PSD95 3'UTR region was highly enriched in Cirbp immunoprecipitated samples compared to $\operatorname{IgG}$ 
controls (Figure 3H). Mechanistically, these finding suggest that Cirbp binds to PSD953'UTR, and post-transcriptionally regulates its expression.

Cirbp up-regulates PSD95 expression and improves hypoxia-induced dendritic spines impairment

To examine whether Cirbp influences the expression of PSD95 to regulate hypoxiainduced structural change and dendritic spines injury, we showed that the protein level of PSD95 was rescued by over-expression of Cirbp following $24 \mathrm{~h}$ of hypoxia-exposure in hippocampal neurons and HT-22 cells (Figure 4A, and Supplementary Figure 2C). Western blot analysis in pEGFP-Cirbp and mCherry-Flag lentivirus infected primary hippocampal neurons showed that overexpressed Cirbp significantly reversed the hypoxia-induced reduction in spine density (Figure 4B-D). These results presented that Cirbp controls PSD95 expression under hypoxia and is a novel key factor in protecting hypoxia-induced dendritic spine morphology abnormalities.

\section{Overexpressed Cirbp protects hypoxia-induced cognitive capacity dysfunction}

Further, we were interested whether over-expression of Cirbp could rescue the cognitive capacity defect in hypoxia exposed mice. AAV-Cirbp-GFP was injected into the hippocampal CA1 region to establish the Cirbp expressing in vivo model. Fluorescence imaging showed that the spontaneous GFP-fluorescence was localized in the hippocampal CA1 region (Figure 5A). The blot results showed that Cirbp expression was increased in the hippocampus, and that hypoxia-induced PSD95 downregulation was rescued upon hypoxic exposure (Figure 5B). Furthermore, MWM and SIAT results revealed that over-expressing Cirbp in the hippocampus reversed the hypoxia-induced memory impairment, with decreased escape latency (Figure 5C, D), and increased step-down time (Figure 5H). The NORT data showed that there was no significant difference in total distance and the mean speed among the mice in these groups (AAV-Con Normoxia, AAV-Con Hypoxia, AAV-Cirbp Normoxia and AAVCirbp Hypoxia) (Supplementary Figure 2D). However, the exploring time for new objects (Figure 5G) and the discrimination index (DI) (Figure 5F) in AAV-Cirbp Hypoxia group were significantly greater than in the AAV-Con Hypoxia group. These results suggested that over-expression of Cirbp retrieved memory damage caused by 
hypoxic exposure in mice.

Moreover, Golgi staining showed that there was no significant change in dendritic branches in hippocampal CA1 region in each of the groups (Figure 6A-C). Under hypoxic condition, Cirbp overexpression significantly reversed the reduction in the density in basal dendrites and apical dendrites (Figure 6D-F), and decreased the length of spines' necks (Figure 6G). These findings corroborated that over-expression of Cirbp attenuated the hypoxia-induced dendritic spine plasticity disorder (Figure 6H).

\section{Discussion}

The effect of chronic hypobaric hypoxia on the central nervous system is multifaceted, including impaired cerebral auto-regulation, gliovascular deficit, oxidative stress, and neuroinflammation, ultimately converting into cognitive deficits (13). Abnormal structure of dendritic spines is a common phenomenon in central nervous system disorders $(14,15)$. Synaptic proteins are often associated with disease neuropathology, but their role in the etiology of hypoxia induced cognitive function impairment is not fully understood. In this study, we examined long-term hypobaric hypoxic exposure on cognitive capacity, dendritic arborization of neuron and spine number in hippocampus region of brain which plays an important role in processing and remembering learned information. It was observed that mice exposed to hypoxia exhibited hippocampal mediated memory deficits with accompanying abnormal neuronal spines morphology. Furthermore, we established that the level of synaptic protein PSD95 was suppressed by hypoxic exposure, and that overexpression of PSD95 mitigated the ill-effects of hypoxia on cognitional dysfunction and dendritic spine abnormities. Biochemical and molecular analysis indicated that the level of PSD95 was post-transcriptionally regulated at the RNA level. By analyzing RNA binding protein motif, we found that there are several Cirbp binding sites in 3'UTR region of PSD95 mRNA and Cirbp regulates PSD95's expression via binding with its 3'UTR region. Finally, abnormal cognitive performance and dendritic spine density under hypoxia were partially rescued after expression of Cirbp. Collectively, these results delineate a Cirbp-PSD-95 axis in the hippocampus that rivals dendritic spine remodeling and cognitive impairment in hypoxia exposed mice (Figure 6H). 
As the main environmental characteristics of the plateau, hypobaric hypoxia has attracted more attention to the effects and mechanisms of human health. In addition to the respiratory and circulatory system, CNS is also a sensitive target organ for hypoxiainduced injuries. Long-term hypoxia stress leads to attention deficit, memory loss, and cognitive dysfunction, even increased risk of neurodegenerative diseases, such as Alzheimer's disease (AD) (16-18). Dendritic spines are small protrusion emerging from the dendritic shaft in many neurons, and represent the postsynaptic element of most excitatory glutamatergic synapses. These are highly dynamic structures, and changes in the number, size and shape of spines are correlated with modifications in synaptic strength (19). An exuberant excess of spines having an immature, long and thin morphology is a well described phenotype related with mental impairment (20). The hippocampus is primarily responsible for learning-memory process, and its CA1 region is closely related to short-term memory and spatial memory, and is the most sensitive to hypoxia-induced damage $(2,21,22)$. Synaptic plasticity in the hippocampus is an established neurobiological basis for learning and memory. Our studies showed that hypoxia-exposed animals had deficits in memory extraction and presentation, consistent with impaired learning and memory. Further studies indicated that the hypoxic exposure disturbed dendritic spines morphology balance, with lower stable dendritic spines and more immature dendritic spines. Previous studies have confirmed the positive relationship between dendritic spine morphology and synaptic functions (23). And stable dendritic spines can enhance brain memory storage capacity by establishing new synaptic connections(24). The formation or enlargement of dendritic spines affects the enhancement of synaptic connections, while the shrinking or disappearance of dendritic spines represents a weakening of synaptic connections (25). RBPs have been identified as key molecules in many diseases including neurodegenerative disorders (26). Cirbp as a cell stress molecule senses changes in the external environment including cold, hypoxia, and ultraviolet light and regulates the expression of related molecules in the cell (6). Importantly, Cirbp has been shown to play a positive role in hypothermia-induced neuroprotection(27), sleep homeostasis (28), circadian(29), cardioprotection (7), inflammatory responses of shock and 
sepsis(30) and neonatal brain injury(31). However, most of the research on Cirbp has focused on its role upon cold exposure, and its function in hypoxia has yet to be delineated. A previous study has confirmed a protective role for Cirbp in hypoxiainduced nerve injury (9), consistent with our experiment. Our novel findings are the first to demonstrate that Cirbp post-transcriptionally regulates PSD95 expression to affect hypoxia-induced dendritic spine remodeling defect. These findings shed light on the important post-transcriptional regulatory role of RNA-binding proteins in controlling dendritic spine homeostasis and learning-memory ability. In our unpublished work, we found that Cirbp, most located in nucleus, interacted with several ribosomal proteins and translation initiation factors, suggesting Cirbp has high propensity to affect RNA processing, nuclear translocation, and translational efficiency. The detail mechanisms are clearly worthy of further study.

PSD95, as the main elements of chemical synapses, interacts with glutamate receptors, cell adhesion molecules and cytoskeleton elements. As it is well known, PSD95 can modulate the stability of basal synapses and the activity-dependent structural plasticity of PSD and excitatory chemical synapses caused thereby (32). Previous studies have shown that PSD95 was post-transcriptionally regulated at excitatory synapses and there are several neurological disorders, including $\mathrm{AD}$, in which impairments in the normal function of PSD95 are associated with posttranslational modifications (33). PSD95 is also a target of several signaling pathways that induce posttranslational modifications, including palmitoylation, phosphorylation, ubiquitination, nitrosylation, and neddylation. These modifications determine the synaptic stability and function of PSD95, and thus regulate the fates of individual dendritic spines in the nervous system (34). Moreover, PSD95 has been shown to facilitate posttranslational modifications as to modulate its postsynaptic localization within the dendritic spine, thereby influencing chemical synaptic transmission regulation in the CNS (34). The expression of PSD95 is controlled and restricted by at least two mechanisms: the action of miR-125a and the degradation of PSD95 mRNA, the latter of which is mediated by two polypyrimidine tract binding (PTB) proteins. The overall result is inhibition of excitatory synapse formation as well 
as neuronal maturation (35). The synaptic localization of PSD95 can be regulated by various posttranslational modifications, depending on developmental stage, synaptic activity, and disease. Here we found that PSD95 was significantly reduced at its Protein level absence a change in its RNA level. And there was no difference in PSD95 degradation rates under hypoxic and normoxic conditions. Thus, these findings suggested that post-transcriptional regulation determines the expression of PSD95. RNA-binding protein (RBP) plays a center role in post-transcriptional regulation, and we find RNA binding protein Cirbp binds PSD95 mRNA and regulates its expression.

Combined, the present data offer novel understanding of regulatory molecules of cognitive dysfunction under long-term exposure to high altitude environment. Our data demonstrate that Cirbp alleviates hypoxia-induced learning-memory impairment, and Cirbp binds PSD95 RNA, forming a novel signaling pathway to balance dendritic spine remodeling. Thus, Cirbp-PSD95 axis acts as a key protective regulator in hypoxic stress, offering a potential new therapeutic site in mitigating the aberrant effects of hypoxia.

\section{Materials and methods}

Animals and Hypoxic Exposure

All procedures involving animals were in accordance with the procedures outlined in the "National Institutes of Health guide for the care and use of Laboratory animals, Eighth Edition" (https://grants.nih.gov/grants/olaw/Guide-for-the-Care-and-use-oflaboratory-animals.pdf), and were approved by the Institutional Animal Care and Use Committee of the Fourth Military Medical University (01 Mar 2017). 8-week C57BL/6 mice were obtained from the animal center of the Fourth Military Medical University and were kept under constant temperature $\left(22-24^{\circ} \mathrm{C}\right)$ and $12 \mathrm{~h}$ light-dark cycle with free access to food and water. Mice were randomly assigned into two groups (normoxia and hypoxia). After five days of adaptation, the normoxic (control) group was kept at normal atmospheric pressure, and the hypoxic group was placed in were placed in the decompression chamber (Fenglei Co. Ltd., China) for continuous hypobaric hypoxic exposure equal to an altitude of $6000 \mathrm{~m}$ (barometric pressure $=349 \mathrm{mmHg}$ and partial $\mathrm{O}_{2}$ pressure $=8-9 \%$ ) for 14 days as described in our previous report (36). 


\section{Cell Culture and Hypoxia Exposure}

Primary hippocampal neurons were cultured as previously described (37). Embryonic day 18 C57BL/6 mice embryos were placed in pre-cooled HBSS Hanks (Gibco, USA) and stripped off the meninges carefully under stereo-microscope (Nikon, Japan). Hippocampi were isolated by removing the bleeding spots, and treated with trypsin (12604013, Gibco, USA). Hippocampal dissociated cells were cultured in Neurobasal medium (Gibco, USA) with B27 (17504044, Gibco, USA), L-Glutamine (G6392-1VL, Sigma-Aldrich, USA), streptomycin and penicillin (MP Biomedicals, USA), and half-liquid exchange was performed every 3 days. For assessment of dentric spines, neurons were infected with p-EGFP lentivirus (Obio, China). All experiments were conducted in compliance with the Guide for the Care and Use of Laboratory Animals and approved by the Institutional Animal Care and Use Committee of the Air Force Military Medical University. Mouse hippocampal neuronal HT-22 cells were cultured as detailed in our previous study (38).

Cells were exposed to hypoxia in a humidified microaerophilic incubation system (DWS-H85, DonWhitley, UK) filled with $1 \% \mathrm{O}_{2}$ and $5 \% \mathrm{CO}_{2}$ as described in previous studies $(9,36)$. Culture medium was placed in the hypoxia incubator for more than $9 \mathrm{~h}$ in advance.

\section{Morris Water Maze (MWM) Test}

Animals were trained in the hidden platform version of the MWM task as described in our publications (39).The hidden platform training was performed for 5 days straight (4 trials per mouse, per day). Each animal was placed in one of four chosen starting locations in the pool and had $60 \mathrm{~s}$ to locate the hidden platform. If an animal failed to find the platform, it was placed on top of it and had to remain there for $20 \mathrm{~s}$ before being put back into its cage by the experimenter. After 5 days of training, one probe trial was performed per mice and each mouse searched for $60 \mathrm{~s}$. All trials were recorded via a digital camera connected to Enthovision ET software and DigiBehave system (Xinruan, China) which was used to record the animals' behaviors.

Novel Object Recognition Test (NORT)

The novel object recognition protocol was developed based on previous procedures 
(40). After 30 minutes room acclimation, mice were placed individually in open field enclosure and allowed to explore the empty environment. Then mice were placed in the open field enclosures $(50 \mathrm{~mm} \times 35 \mathrm{~mm} \times 20 \mathrm{~mm})$ in which two identical objects (A1 and A2) were placed equidistant from the corners of the enclosure, allowed 3 minutes to explore freely. On the second day, one of the A objects was replaced with B object before 3 minutes exploration. The familial object exploration time $\left(\mathrm{T}_{\mathrm{A}}\right)$ and novel object exploration time $\left(\mathrm{T}_{\mathrm{B}}\right)$ were recorded on the video tracking system (DigiBehave system, Xinruan) and discriminate index $(\mathrm{DI})$ was calculated $\left[\mathrm{DI}=\left(\mathrm{T}_{\mathrm{B}}-\mathrm{T}_{\mathrm{A}}\right) /\left(\mathrm{T}_{\mathrm{B}}+\mathrm{T}_{\mathrm{A}}\right)\right]$. The area was wiped clean with $70 \%$ ethanol between each test to minimize residual odor cues effect.

\section{Step-down Inhibitory Avoidance Test (SIAT)}

The step down inhibitory avoidance test was performed following previous protocol (41). During the training session, the animals were placed on a circular platform and received a $2 \mathrm{~s}, 0.4 \mathrm{~mA}$ scrambled foot shock right after stepping down onto the grid with all 4 paws. Mice were trained for three times and tested after $2 \mathrm{~h}$ interval. If the animal did not step down the platform after $150 \mathrm{~s}$, the final time was recorded as $150 \mathrm{~s}$.

\section{Golgi Staining and Morphological Analysis}

For Golgi staining as our previous description (42), mice were deeply anesthetized with sodium pentobarbital (100 mg/kg, i.p.) and transcardially perfused with $0.9 \%$ saline. Whole brains were processed using an FD Rapid GolgiStain ${ }^{\mathrm{TM}} \mathrm{Kit}(\mathrm{A}: \mathrm{B}=1: 1)$ (FD Neuro Tech-Inc, USA) and sliced at $100 \mu \mathrm{m}$ using a vibratome (VT1000S, Leica). Sections were collected on gelatinized glass slides and immersed in Golgi solution in the dark at room temperature for $15 \mathrm{~min}$. The tissue sections were dehydrated with $50 \%$, $75 \%, 95 \%$, and $100 \%$ ethanol for 4 min each. The slides were cleared with xylene (Fuyu, China) at room temperature in the dark, and coverslip with DPX Mountant (SigmaAldrich, USA). An optical microscope (Zeiss, Germany) was used to image the slides, with low magnification (20X) for dendritic tree and high magnification (100X) for spine reconstruction. Quantitative differences in the density of dendritic spines on individual dendritic branch orders between conditions was determined as previously 
described (42).

RNA Extraction and Real-time quantitative PCR

Total RNA were extracted with Trizol reagent (Invitrogen, USA), and cDNAs were synthesized with the PrimeScript ${ }^{\mathrm{TM}}$ RT Master Mix (RR036A, TAKARA, Japan). Realtime quantitative PCR was performed using TB Green ${ }^{\circledR}$ Premix Ex Taq $^{\mathrm{TM}}$ II assays (820A, TAKARA, Japan) in FAST-7500 system (Applied Biosystems, USA) following standard protocols (39). The Mus musculus PSD95 cDNA fragment were amplified by primers 5'-TGC ATC TCT GCG AAG CAA CC-3' and 5'-GCG TCA ATT ACA TGA AGC ACA TCC-3'), and two oligonucleotides 5'-AGC CAT GTA CGT AGC CAT CC3'and 5'-GCT GTG GTG GTG AAG CTG TA-3' were used as the specific primers to amplify Mus musculus $\beta$-actin cDNA as a loading control. . The $2^{\Delta \Delta \mathrm{Ct}}$ values were calculated (43) and data were normalized to $\beta$-actin.

\section{Plasmid construction and transfection}

Cirbp plasmid (pLenti-EF1a-EGFP-P2A-Puro-CMV-Cirbp-3Flag) and pEGFP-N2 control vector were constructed by Obio Technology Company (Shanghai, China). Transfection complexes, consisting of $5 \mu \mathrm{g}$ plasmid DNA and $10 \mu \mathrm{l}$ Lipofectamine reagent (Thermo Fisher Scientific) were added to the $6 \mathrm{~cm}$ wells in Opti-MEM ${ }^{\circledR}$ Medium (Invitrogen, USA). Cells were replaced with the DMEM-High Glucose (Gibco, USA) after $6 \mathrm{~h}$ and the follow-up experiments continued.

In vitro Lentivirus infection

Primary hippocampal neurons were seeded in cell dishes covered by polylysine at a density of $2 \times 10^{5}$, cultured for three days for semi-liquid exchange, and directly added to the virus solution with pLenti-CMV-EGPF-3FLAG (Obio Technology, China) or pLenti-CMV-mCherry-3FLAG (Obio Technology, China) and pHBLV-CMV-MCSEF1-Zsgreen1-T2A-puro (Hanbio Biotechnology, China) on the fifth day according to the dose of $\mathrm{MOI}=10$. (Virus amount=cell number $\times$ MOI value $/$ original virus titer $) \times$ $10^{3}$. The following day, the cell liquid was exchanged with fresh solution, and then continued to culture and keep changing every three days.

\section{Protein Extraction and Western Blot Analysis}

Western blot was conducted as previously described (44). The following antibodies 
were used for the specific detection of individual protein signals: Cirbp, diluted 1:500, Proteintech10209-2-AP (UK); PSD95, 1:1000, Abcam18258, (UK); $\beta$-actin, 1:1000, Sigma A5441, (UK). The protein bands were visualized by ELC plus chemiluminescence (Qicai, China) and its intensities were determined by Image $\mathbf{J}$ software (National Institutes of Health, MD). Each protein band was normalized to $\beta$ actin values and expressed as the relative intensity ratio.

Stereotactic injection of virus

The anesthetized C57BL/6 mice were mounted into the stereotaxic frame (RWD Life science, China). Needle was vertically and bilaterally lowered into the hippocampus (ML: \pm 1.5 ; AP: -2.18 ; DV: $-1.6 \mathrm{~mm}$ from bregma and skull). (0.3 $\mu \mathrm{l}$ $\left(1 \times 10^{12} \mathrm{VG} / \mathrm{ml}\right)$ of adeno-associated virus (AAV-CIRBP: pAOV-SYN-EGFP-P2ACIRBP; AAV-PSD95: AAV-SYN-EGFP-P2A-Dlg-3FLAG) (Obio, Shanghai, China) was then bilaterally infused for $3 \mathrm{~min}$ and another $5 \mathrm{~min}$ allowed for diffusion before the injector was raised (45). At the completion of viral infection, the incised skin covering the skull was grabbed with surgical staplers and animals were placed in a constant temperature heater at $32^{\circ} \mathrm{C}$ to rewarm. Next, rats were returned to their home cages for 1-week recovery period. AAV viruses were expressed and detected after 3 weeks.

\section{Tissue Fluorescence Staining}

At the end of the experiments, the animals were deeply anesthetized and transcardially perfused with $4 \%$ cold paraformaldehyde in $0.1 \mathrm{M}$ phosphate buffer $(\mathrm{PB})$. Brains were dissected out and post-fixed in the $30 \%$ sucrose solution for 3 days. Mice brains were sliced from the coronal part of the standard hippocampus (thickness $20 \mu \mathrm{m}$ ). 0.1M PBS and 0.3\% Triton-X100 (Kehao, China) was used for diluting all immunoreagents. Then, they were blocked with 3\% BSA (MP Biomedicals, USA) for $30 \mathrm{~min}$ and dripped in primary antibody (NeuN, abcam104224, UK) overnight at $4{ }^{\circ} \mathrm{C}$, subsequently incubated with secondary antibodies conjugated with a fluorescent dye for 2 h. After washing, these sections were incubated with 4',6-diamidino-2phenylindole (DAPI, Beyotime, China) for $30 \mathrm{~min}$ at room temperature, and then covered with glycerin. Immunofluorescent images were captured on a fluorescence 
microscope (Zeiss, Germany).

\section{Luciferase Reporter Activity Experiment}

Three fragments of mouse PSD95 (dlg4) mRNA 3'UTR [3'UTR-A(2491-2769nt), 3'UTR-B(2770-3048nt) and 3'UTR-C(3049-3327nt)] were cloned into pMIR-Report luciferase plasmid (GeneChem, China). For luciferase reporter assays, HT-22 cells were co-transfected with pMIR-REPORT luciferase plasmid with PSD95 3'UTR-A, -B and -C (500 ng/well), Cirbp plasmids (500 ng/well) or NC (control) plasmids (500 ng/well), and Prl-TK plasmid (50 ng/well) using Lipofectamine 2000 (Invitrogen, USA). The luciferase activity was determined with the Dual-Luciferase Reporter Assay System according to the manufacturer's instructions as described (46).

\section{RNA Binding Protein Immunoprecipitation (RIP)}

Cell samples collected were lysed with an equal volume of complete RIP lysate buffer $(100 \mu \mathrm{l}$ RIP lysis buffer, $0.5 \mu$ protease inhibitor mixture, $0.25 \mathrm{ul}$ RNA inhibitor /unit). Cirbp antibody (5 $\mu \mathrm{g})$ or $\operatorname{IgG}$ antibody (control) mixed with protein $\mathrm{A} / \mathrm{G}$ Sepharose (Santa Cruz Biotechnology, Dallas TX) and continuously cultured at room temperature for $30 \mathrm{~min}$. The cell lysate was added to a RIP immunoprecipitation buffer (RIP wash buffer, 0.5M EDTA, ribonuclease inhibitor) containing a magnetic beadantibody complex to obtain a mixed solution, which incubated at $4{ }^{\circ} \mathrm{C}$ for $9 \mathrm{~h}$. In addition, an aliquot (10\%) of supernatant were signed as Input and another for verification of Western-blot. Then, the immunoprecipitate were resuspended in proteinase $\mathrm{K}$ buffer (RIP wash buffer, $10 \%$ SDS, proteinase $\mathrm{K}$ ) and incubated at $55^{\circ} \mathrm{C}$ for 30 min. RNA was isolated using Trizol reagent and subjected to RT-qPCR, using specific primers to detect the Cirbp binding.

\section{Data Presentation and Statistical Analysis}

All experimental data was analyzed using SPSS 20.0 software (IBM Corp, USA). The data were expressed as mean \pm standard error of the mean (SEM). The results were tested by one-way ANOVA for comparison among three or more groups, while differences between two groups were analyzed with Student's $t$-test. Data from the experiments involving several treatments were analyzed by multi-way ANOVA. A Tukey's post hoc test revealed which groups differed significantly from one other. 
497 Probability values less than 0.05 were considered statistically significant.

498

499

\section{List of abbreviations}

500

AD, Alzheimer's disease;

501

ATP, Adenosine triphosphate;

502

CHX, Cycloheximide;

503

Cirbp, Cold-inducible RNA-binding protein;

$504 \quad$ CNS, Central nervous system;

505

DAPI, 4',6-diamidino-2-phenylindole;

506 HA, high altitude;

$507 \quad \mathrm{HH}$, Hypobaric hypoxia;

508 LTP, Long-term potentiation;

509 MWM, Morris water maze;

510 NORT, Novel object recognition test;

511 NR2A, N-methyl-D-aspartate 2A receptors;

$512 \quad$ NR2B, N-methyl-D-aspartate 2B receptors;

513 PSD, Post-synaptic density;

514 RBP, RNA-binding protein;

515 SIAT, Step-down inhibitory avoidance test.

516 


\section{Declarations}

Ethics approval and consent to participate

All procedures involving animals were in accordance with the procedures outlined in the "National Institutes of Health guide for the care and use of Laboratory animals, Eighth Edition", and were approved by the Institutional Animal Care and Use Committee of the Fourth Military Medical University.

Consent for publication

Not applicable.

Availability of data and materials

The datasets used and/or analyzed during the current study are available from the corresponding author on reasonable request.

\section{Competing interests}

The authors have no conflicts of interest to declare that are relevant to the content of this article.

\section{Funding}

This work was supported by Key Project of National Natural Science Foundation of China 81330045 (W.L.), 81730053 (W.L.); National Natural Science Foundation of China 81402650 (Z.C.), 81673121 (W.L.); Program of Shaanxi Science and Technology Innovation Team 2018TD-001 (W.L.).

\section{Authors' contributions}

YZ performed experiments, designed experiments, analyzed data, prepared early figure versions, figure legends, and wrote the manuscript. HL performed biochemistry experiments, analyzed data, analyzed data, prepared figures and figure legends. YL performed experiments, analyzed data. ZZ performed Golgi staining and morphological analysis. QZ performed experiments. CX performed the stereotactic injection of virus. YZ helped in animal exposure experiments and behavioral studies. ZC directed research, designed experiments and was a major contributor in writing the manuscript. WL designed experiments, directed research and revised the manuscript. All authors read and approved the final manuscript. 


\section{References}

1. West JB. Are Permanent Residents of High Altitude Fully Adapted to Their Hypoxic Environment? High altitude medicine \& biology. 2017;18(2):135-9.

2. Chauhan G, Roy K, Kumar G, Kumari P, Alam S, Kishore K, et al. Distinct influence of COX-1 and COX-2 on neuroinflammatory response and associated cognitive deficits during high altitude hypoxia. Neuropharmacology. 2019;146:138-48. 3. Yu L, Chen Y, Wang W, Xiao Z, Hong Y. Multi-Vitamin B Supplementation Reverses Hypoxia-Induced Tau Hyperphosphorylation and Improves Memory Function in Adult Mice. Journal of Alzheimer's disease : JAD. 2016;54(1):297-306.

4. Wang J, Ke T, Zhang X, Chen Y, Liu M, Chen J, et al. Effects of acetazolamide on cognitive performance during high-altitude exposure. Neurotoxicology and teratology. $2013 ; 35: 28-33$.

5. Chen X, Zhang Q, Wang J, Liu J, Zhang W, Qi S, et al. Cognitive and neuroimaging changes in healthy immigrants upon relocation to a high altitude: A panel study. Human brain mapping. 2017;38(8):3865-77.

6. Liao Y, Tong L, Tang L. The role of cold-inducible RNA binding protein in cell stress response. 2017;141(11):2164-73.

7. Liu Y, Xing J. Chronic hypoxia-induced Cirbp hypermethylation attenuates hypothermic cardioprotection via down-regulation of ubiquinone biosynthesis. 2019;11(489).

8. Zhu X, Buhrer C, Wellmann S. Cold-inducible proteins CIRP and RBM3, a unique couple with activities far beyond the cold. Cellular and molecular life sciences : CMLS. 2016;73(20):3839-59.

9. Zhang Q, Wang YZ, Zhang W, Chen X, Wang J, Chen J, et al. Involvement of Cold Inducible RNA-Binding Protein in Severe Hypoxia-Induced Growth Arrest of Neural Stem Cells In Vitro. Molecular neurobiology. 2017;54(3):2143-53.

10. Gipson CD, Olive MF. Structural and functional plasticity of dendritic spines - root or result of behavior? Genes, brain, and behavior. 2017;16(1):101-17.

11. Berry KP, Nedivi E. Spine Dynamics: Are They All the Same? Neuron. 2017;96(1):43-55. 
577 12. Sephton CF, Yu G. The function of RNA-binding proteins at the synapse: 578 implications for neurodegeneration. Cellular and molecular life sciences : CMLS. $579 \quad 2015 ; 72(19): 3621-35$.

580 13. Wilson $\mathrm{MH}$, Newman S, Imray CH. The cerebral effects of ascent to high altitudes. 581 The Lancet Neurology. 2009;8(2):175-91.

582 14. Fiala JC, Spacek J, Harris KM. Dendritic spine pathology: cause or consequence 583 of neurological disorders? Brain research Brain research reviews. 2002;39(1):29-54.

584 15. Nishiyama J. Plasticity of dendritic spines: Molecular function and dysfunction in 585 neurodevelopmental disorders. Psychiatry and clinical neurosciences. 2019;73(9):54158650.

16. Gao YX, Li P, Jiang CH, Liu C, Chen Y, Chen L, et al. Psychological and cognitive 588 impairment of long-term migrators to high altitudes and the relationship to 589 physiological and biochemical changes. European journal of neurology. $590 \quad 2015 ; 22(10): 1363-9$.

591 17. Snyder B, Duong P, Trieu J, Cunningham RL. Androgens modulate chronic 592 intermittent hypoxia effects on brain and behavior. Hormones and behavior. $593 \quad 2018 ; 106: 62-73$.

594 18. Yaffe K, Laffan AM, Harrison SL, Redline S, Spira AP, Ensrud KE, et al. Sleep595 disordered breathing, hypoxia, and risk of mild cognitive impairment and dementia in 596 older women. Jama. 2011;306(6):613-9.

597 19. Tada T, Sheng M. Molecular mechanisms of dendritic spine morphogenesis. 598 Current opinion in neurobiology. 2006;16(1):95-101.

599 20. Stein IS, Zito K. Dendritic Spine Elimination: Molecular Mechanisms and 600 Implications. The Neuroscientist : a review journal bringing neurobiology, neurology 601 and psychiatry. 2019;25(1):27-47.

602 21. Titus AD, Shankaranarayana Rao BS, Harsha HN, Ramkumar K, Srikumar BN, 603 Singh SB, et al. Hypobaric hypoxia-induced dendritic atrophy of hippocampal neurons 604 is associated with cognitive impairment in adult rats. Neuroscience. 2007;145(1):26560578.

606 22. Kumari P, Kauser H, Wadhwa M, Roy K, Alam S, Sahu S, et al. Hypobaric hypoxia 
607 impairs cued and contextual fear memory in rats. Brain research. 2018;1692:118-33.

608

609

610

611

612

613

614

615

616

617

618

619

620

621

622

623

624

625

626

627

628

629

630

631

632

633

634

635

636

23. Alvarez VA, Sabatini BL. Anatomical and physiological plasticity of dendritic spines. Annual review of neuroscience. 2007;30:79-97.

24. Stepanyants A, Chklovskii DB. Neurogeometry and potential synaptic connectivity. Trends in neurosciences. 2005;28(7):387-94.

25. Murakoshi H, Yasuda R. Postsynaptic signaling during plasticity of dendritic spines. Trends in neurosciences. 2012;35(2):135-43.

26. Maziuk B, Ballance HI, Wolozin B. Dysregulation of RNA Binding Protein Aggregation in Neurodegenerative Disorders. Frontiers in molecular neuroscience. 2017;10:89.

27. Sun YJ, Ma S, Fan B, Wang Y, Wang SR, Li GY. Therapeutic hypothermia protects photoreceptors through activating Cirbp pathway. Neurochemistry international. 2019;126:86-95.

28. Hoekstra MM, Emmenegger Y, Hubbard J, Franken P. Cold-inducible RNAbinding protein (CIRBP) adjusts clock-gene expression and REM-sleep recovery following sleep deprivation. 2019;8.

29. Gotic I, Schibler U. Posttranscriptional mechanisms controlling diurnal gene expression cycles by body temperature rhythms. RNA biology. 2017;14(10):1294-8.

30. Qiang X, Yang WL, Wu R, Zhou M, Jacob A, Dong W, et al. Cold-inducible RNAbinding protein (CIRP) triggers inflammatory responses in hemorrhagic shock and sepsis. Nature medicine. 2013;19(11):1489-95.

31. Jackson TC, Kotermanski SE, Kochanek PM. Infants Uniquely Express High Levels of RBM3 and Other Cold-Adaptive Neuroprotectant Proteins in the Human Brain. Developmental neuroscience. 2018;40(4):325-36.

32. Suzuki E, Kamiya H. PSD-95 regulates synaptic kainate receptors at mouse hippocampal mossy fiber-CA3 synapses. Neuroscience research. 2016;107:14-9.

33. Young FB, Butland SL, Sanders SS, Sutton LM, Hayden MR. Putting proteins in their place: palmitoylation in Huntington disease and other neuropsychiatric diseases. Progress in neurobiology. 2012;97(2):220-38.

34. Vallejo D, Codocedo JF, Inestrosa NC. Posttranslational Modifications Regulate 
the Postsynaptic Localization of PSD-95. Molecular neurobiology. 2017;54(3):175976.

35. Zheng S, Gray EE, Chawla G, Porse BT, O'Dell TJ, Black DL. PSD-95 is posttranscriptionally repressed during early neural development by PTBP1 and PTBP2. Nature neuroscience. 2012;15(3):381-8, s1.

36. Chen X, Liu X, Li B, Zhang Q, Wang J, Zhang W, et al. Cold Inducible RNA Binding Protein Is Involved in Chronic Hypoxia Induced Neuron Apoptosis by DownRegulating HIF-1alpha Expression and Regulated By microRNA-23a. International journal of biological sciences. 2017;13(4):518-31.

37. Beaudoin GM, 3rd, Lee SH, Singh D, Yuan Y, Ng YG, Reichardt LF, et al. Culturing pyramidal neurons from the early postnatal mouse hippocampus and cortex. Nature protocols. 2012;7(9):1741-54.

38. Xue C, Kang B, Su P, Wang D, Zhao F, Zhang J, et al. MicroRNA-106b-5p participates in lead $\left(\mathrm{Pb}^{2+}\right)$-induced cell viability inhibition by targeting XIAP in HT-22 and PC12 cells. Toxicol In Vitro. 2020.

39. An J, Cai T, Che H, Yu T, Cao Z, Liu X, et al. The changes of miRNA expression in rat hippocampus following chronic lead exposure. Toxicology letters. 2014;229(1):158-66.

40. Lueptow LM. Novel Object Recognition Test for the Investigation of Learning and Memory in Mice. Journal of visualized experiments : JoVE. 2017(126).

41. Izquierdo I, Izquierdo LA, Barros DM, Mello e Souza T, de Souza MM, Quevedo J, et al. Differential involvement of cortical receptor mechanisms in working, shortterm and long-term memory. Behavioural pharmacology. 1998;9(5-6):421-7.

42. Zhao ZH, Zheng G, Wang T, Du KJ, Han X, Luo WJ, et al. Low-level Gestational Lead Exposure Alters Dendritic Spine Plasticity in the Hippocampus and Reduces Learning and Memory in Rats. Scientific reports. 2018;8(1):3533.

43. Schmittgen TD, Livak KJ. Analyzing real-time PCR data by the comparative C(T) method. Nature protocols. 2008;3(6):1101-8.

44. Wang D, Zhang J, Jiang W, Cao Z, Zhao F, Cai T, et al. The role of NLRP3-CASP1 in inflammasome-mediated neuroinflammation and autophagy dysfunction in 
667 manganese-induced, hippocampal-dependent impairment of learning and memory 668 ability. Autophagy. 2017;13(5):914-27.

669 45. Naso MF, Tomkowicz B, Perry WL, 3rd, Strohl WR. Adeno-Associated Virus 670 (AAV) as a Vector for Gene Therapy. BioDrugs : clinical immunotherapeutics, 671 biopharmaceuticals and gene therapy. 2017;31(4):317-34.

672 46. Lu H, Ye Z. QKI regulates adipose tissue metabolism by acting as a brake on 673 thermogenesis and promoting obesity. 2020;21(1):e47929.

674

675 
Figure 1

A

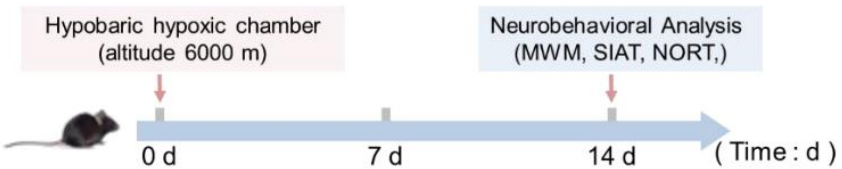

B

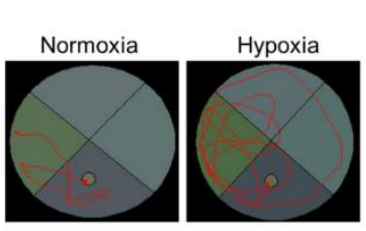

E

C

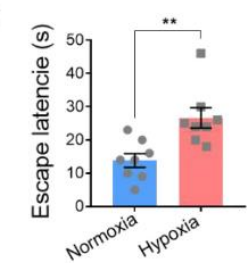

F
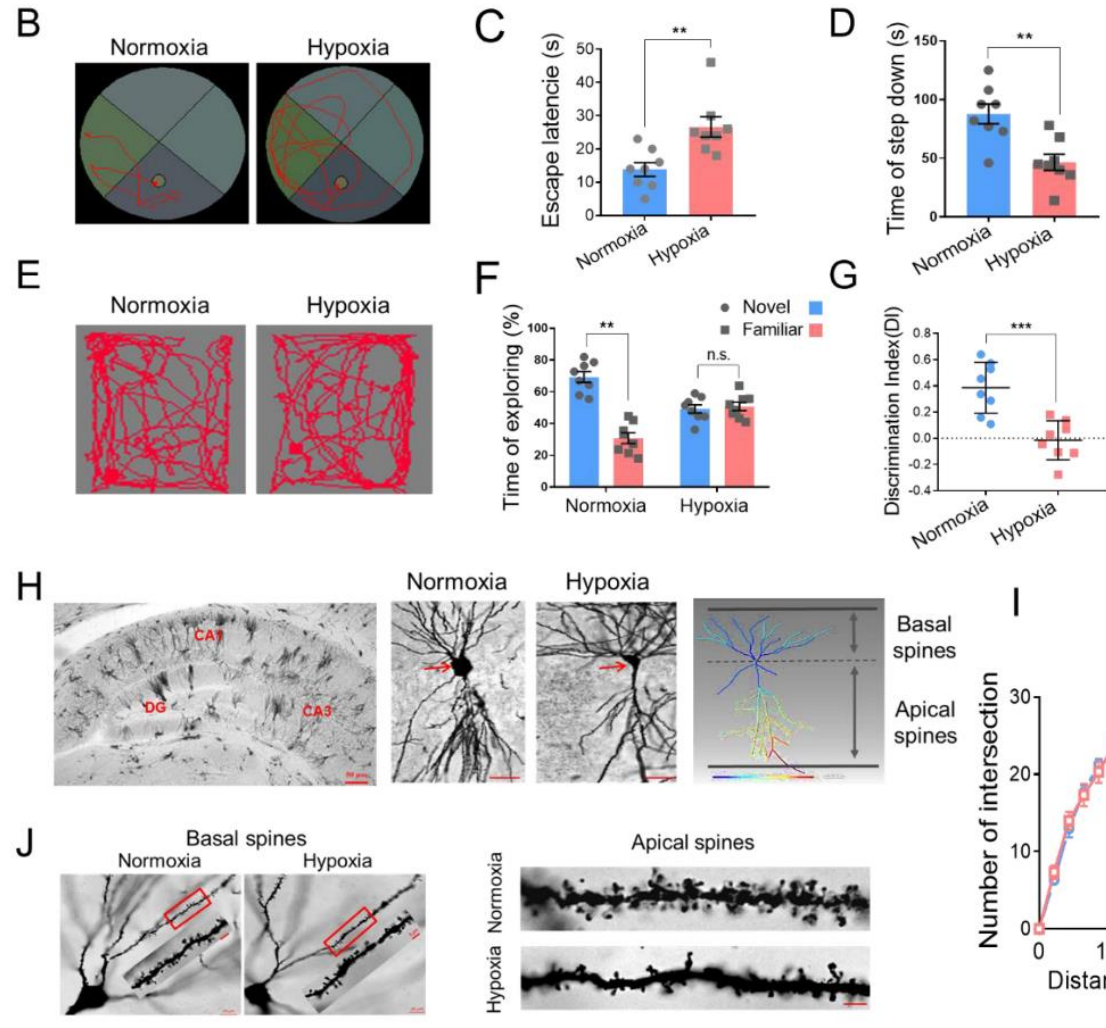

Normoxia Hypoxia
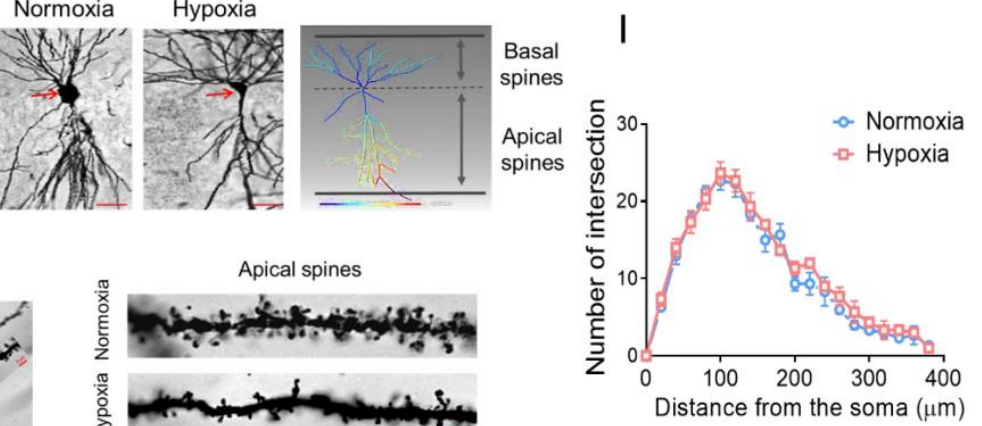

K

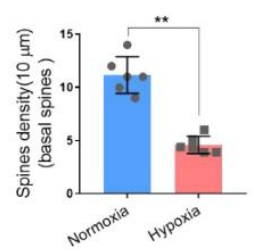

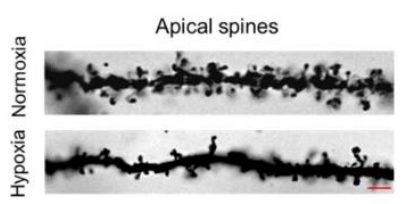

L
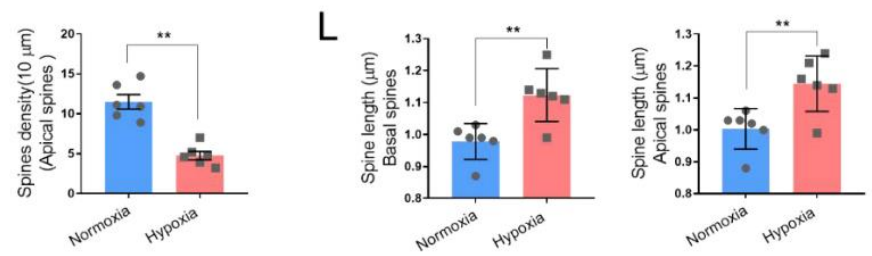

677

678

679

680

681

682

683

684

685

686

Figure 1 Hypobaric hypoxic exposure impaired memory ability and spine morphology.

(A) The scheme of the experimental procedure.

(B-C) Representative locomotion tracking plots (B) and the escape latency (C) of mice in MWM test ( $\mathrm{n}=8$, Student's $t$-test, \pm SEM).

(D) The latency time prior to descent from the platform in SIAT test ( $\mathrm{n}=8$, Student's $t$ test, \pm SEM).

(E-G) Representative locomotion tracking plots (E), exploring time on new objects (F) and the discriminate index $(\mathrm{G})$ in NORT test ( $\mathrm{n}=8$, Student's $t$-test, $\pm \mathrm{SEM})$. 
687 (H) Left: Representative golgi staining of mice hippocampus. DG, dentate gyrus, scale 688 bar $=50 \mu \mathrm{m}$. Middle: Golgi staining of CA1 pyramidal neurons, scale bar $=20 \mu \mathrm{m}$. Right: 689 Imaris reconstruction of CA1 pyramidal neurons.

690 (I) Number of dendritic intersections of reconstructed pyramidal neurons by Sholl 691 analysis (12 neurons/3 mice per group, Student's $t$-test, $\pm \mathrm{SEM}$ ).

692 (J) Left: Representative Golgi staining of basal dendritic spines in CA1, scale bar=20 $693 \mu \mathrm{m}$ (Red frame indicated target area, scale bar=5 $\mu \mathrm{m}$ ). Right: Representative 694 morphology of apical dendritic spines in CA1, scale bar=5 $\mu \mathrm{m}$.

695 (K) Quantification of basal and apical dendritic spines density and columns represent 696 number of spines per $10 \mu \mathrm{m}$ (12 neurons / 3 mice per group, Student's $t$-test, $\pm S E M)$.

697 (L) Quantification of basal and apical neck length (12 neurons / 3 mice per group, 698 Student's $t$-test, \pm SEM).

699 $* p<0.05, * * p<0.01, * * * p<0.001$. 
A

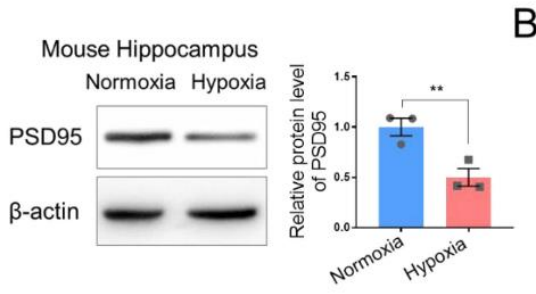

B

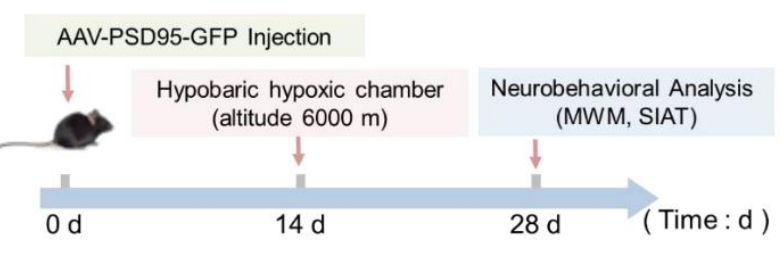

C
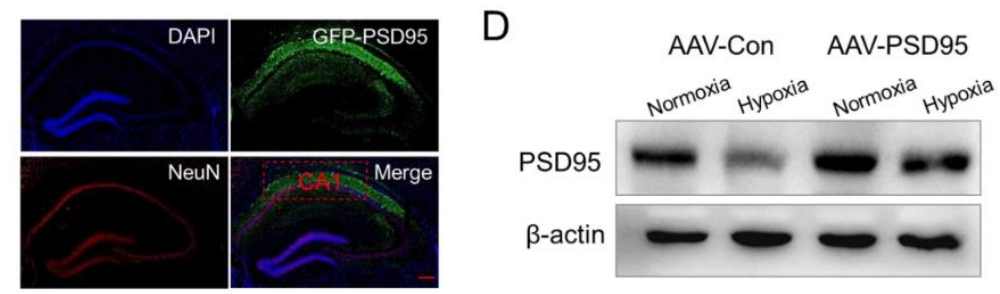

E

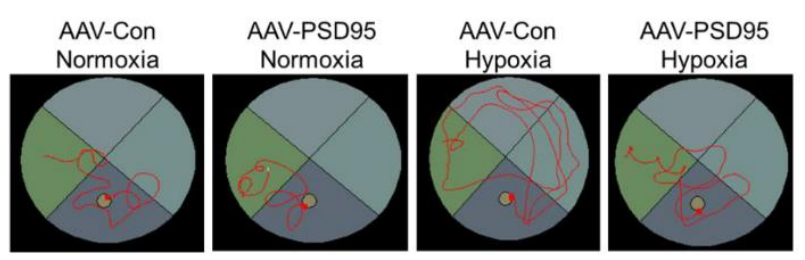

$\mathrm{F}$

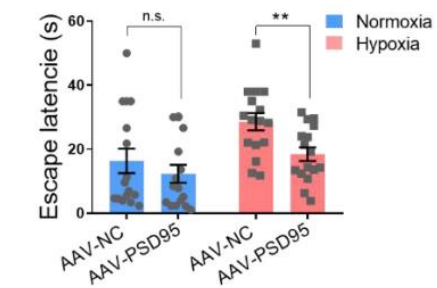

G

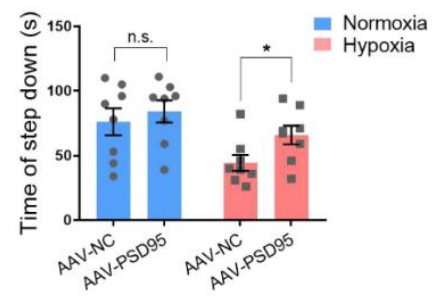

$\mathrm{H}$
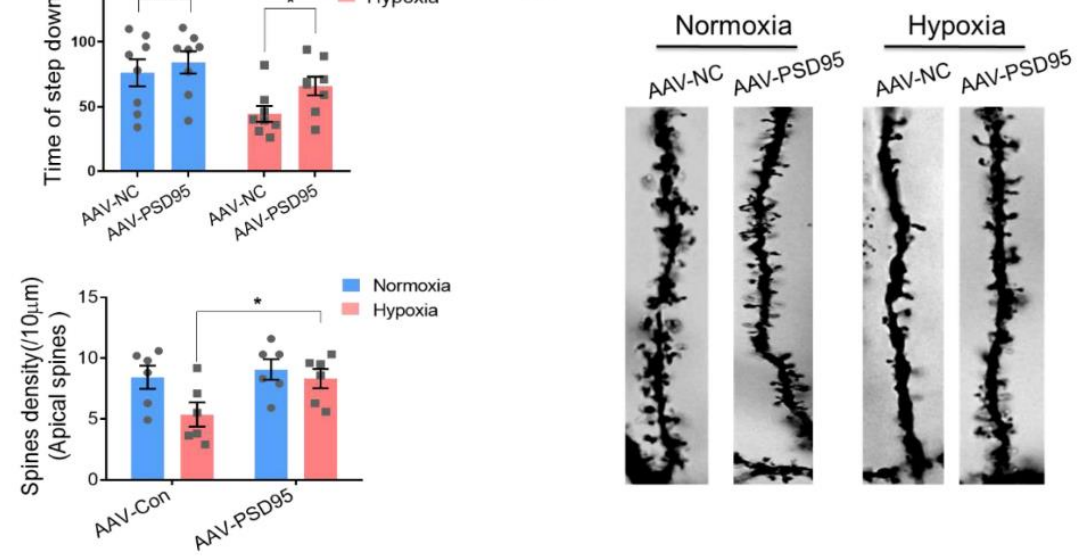

Figure 2 Hypobaric hypoxia down-regulated PSD95 protein level in mice hippocampus and ectopic expression of PSD95 restored memory function and the spine phenotype caused by hypoxic exposure.

(A) Representative immunoblotting and the quantification analysis of PSD95 ( $\mathrm{n}=3$ biological replicates, Student's $t$-test, \pm SEM)

708 (B) Schematic representation of the experimental setup.

709 (C) The fluorescence image of the hippocampus CA1 after stereotacitic injection 710 showing the autofluorescence of AAV-PSD95 (green) and DAPI (blue) and the 
711 immunofluorescence of NeuN (red), scale bar $=50 \mu \mathrm{m}$.

712 (D) Representative immunoblotting of PSD95. ( $\mathrm{n}=3$ biological replicates).

713 (E-F) Representative tracking plots (E) and the escape latency (F) of mice under 714 indicated treatment in MWM test ( $\mathrm{n}=8$, two-way ANOVA, \pm SEM).

715 (G) The latency time of mice under indicated treatment in SIAT test ( $\mathrm{n}=8$, two-way 716 ANOVA, \pm SEM).

717 (H-I) Representative Golgi staining morphology $(\mathrm{H}$, scale bar=5 $\mu \mathrm{m})$ and quantitative 718 analysis of density (I) of apical spines in hippocampus CA1 neurons of mice under 719 indicated treatment (12 neurons / 3 mice per group, two-way ANOVA, \pm SEM) 720 n.s., no significant, $* p<0.05, * * p<0.01$. 


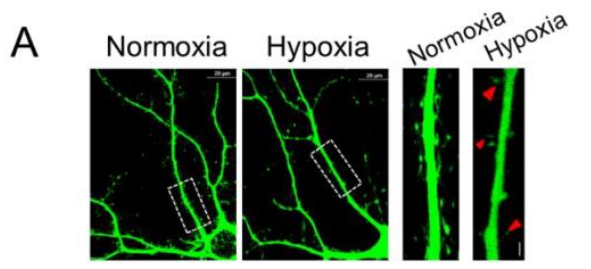

B

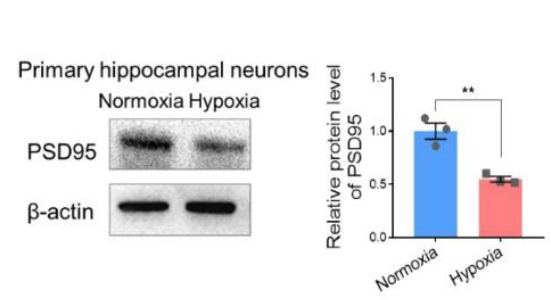

$\mathrm{E}$

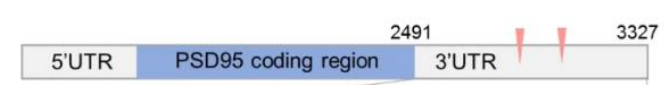

TICCTGCCCTGGCTTGGCCTGGACTCACCCTGCCTCCACCACCTGGGCCCTTG

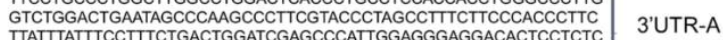
TTATTATTCCTITCTGACTGGATTGAGCCCATTGGAGGGAGGACACTCCTCTC
TGCATGTATCCCTGCACCCCAGATCAGGGCCCCTAGACCCCAGGAACCTGGGG
$2491 \ldots-2769$ TCTGGGGGGGAGCTGGGCTCCTGGTTCCGAGCCCTTGCTCCTTAAGACTCCTA CCCCTGCCCGCCCCCATGCACACACAGACCCACTGGGGGCTCTGCCCTCCC CATTTTCTCCCACACACATTCCAGAAGTCAGGGCCCCTCTCAAGGAGTACCCGC TGTAGGGATGCAGGGCCACAGGCTTCCGCGCTCTCCCGAGGCAGGGGCTGGG
GTCACCCCTGCCCCATCATAACTCCCCACGCCGTTTGAGTTCTCCTTIATTTTCT GTACCCCTGCCCCATCATAACTCCCCACGCCGTTTTGATTCTCCTTATTTTCT
CCATTCTITCTTCTCAAAGGTGGTITTGGGGGGAGAAGCAGGGGGGCTCTC CTGAGGGTCCCCCCGTCTITCACACACCTCCCACCTIIIITCTITGCCGGTTTG CATGAGTGGAAGGTCTAACTGTGGCTTTTTITTTTTCTGGGATTTTATTTCATTTT ATTTGATTTTATTITITTGGGAAAAGGGGAGGGATGGGTCTGGGGAGTGGGGAA TGTGGGAGGGGGGTGGGGGGGCAAAGGTCAGGGGTTGGGTGTCCGGGAGC GAGAGAGTGAAAGGAAGAGACAGACACTTGAAAAAAAAAAAAAAA

G
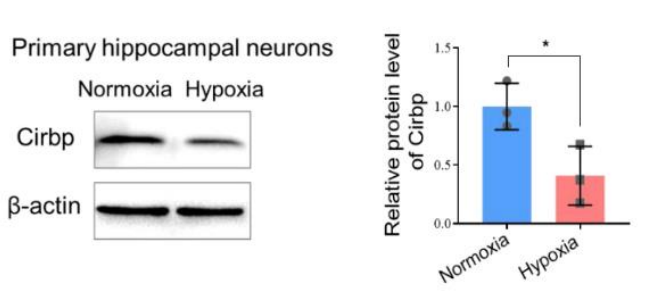
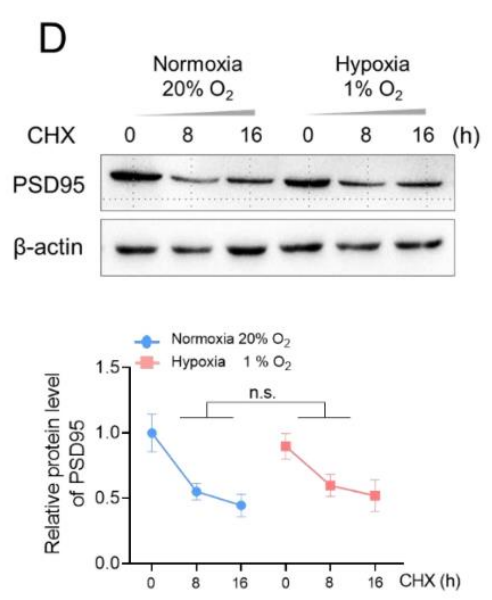

F
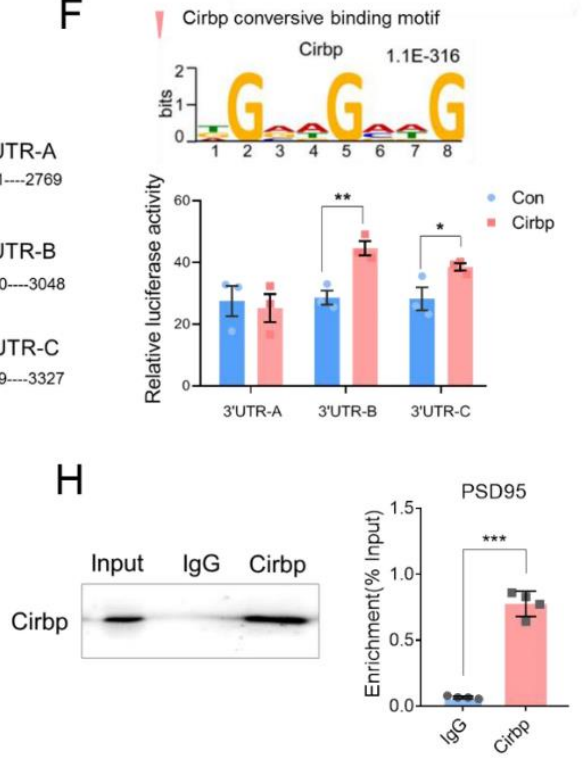

Figure 3 Hypoxic exposure resulted in aberrant dendritic spine morphology and binding.

(A) Left: representative images of hippocampal neurons with hypoxia or normoxia exposure, scale bar $=10 \mu \mathrm{m}$. Middle: enlarged images of hippocampal neurons, red triangle indicated the elimination of spines or filopodia, scale bar $=5 \mu \mathrm{m}$. Right, the quantification of spines density (12 neurons / 3 mice per group, Student's $t$-test, \pm SEM).

(B) Representative immunoblotting (left) and quantitative analysis (right) of PSD95 in primary hippocampal neurons under hypoxic exposure $(n=3$ biological replicates, Student's $t$-test, \pm SEM) 
735 (C) The relative mRNA levels of PSD95 in primary hippocampal neurons under 736 hypoxic exposure ( $\mathrm{n}=3$ biological replicates, Student's $t$-test, \pm SEM).

737 (D) PSD95 expression after CHX treatment based on western blot and quantitative 738 analysis ( $\mathrm{n}=3$ biological replicates, paired Student's $t$-test, \pm SEM).

739 (E) RBP binding motif distributing in PSD95 3'UTR and construction of a series of 740 luciferase reporter vectors containing PSD95 3'UTR fragments.

741 (F) Luciferase activity assays to examine the functional RBP motif sites in the PSD95

742 3'UTR regulated by Cirbp ( $\mathrm{n}=3$ biological replicates, Student's $t$-test, \pm SEM).

743 (G) Representative immunoblotting (left) and the quantitative analysis (right) of Cirbp 744 in primary hippocampal neurons after hypoxia exposure $(n=3$ biological replicates, 745 Student's $t$-test, \pm SEM).

746 (H) RNA immunoprecipitation (RIP) followed by RT-PCR in HT-22 cells ( $\mathrm{n}=4$ 747 biological replicates, Student's $t$-test, \pm SEM). $* p<0.05, * * p<0.01, * * * p<0.001$. 
A Primary hippocampal neurons
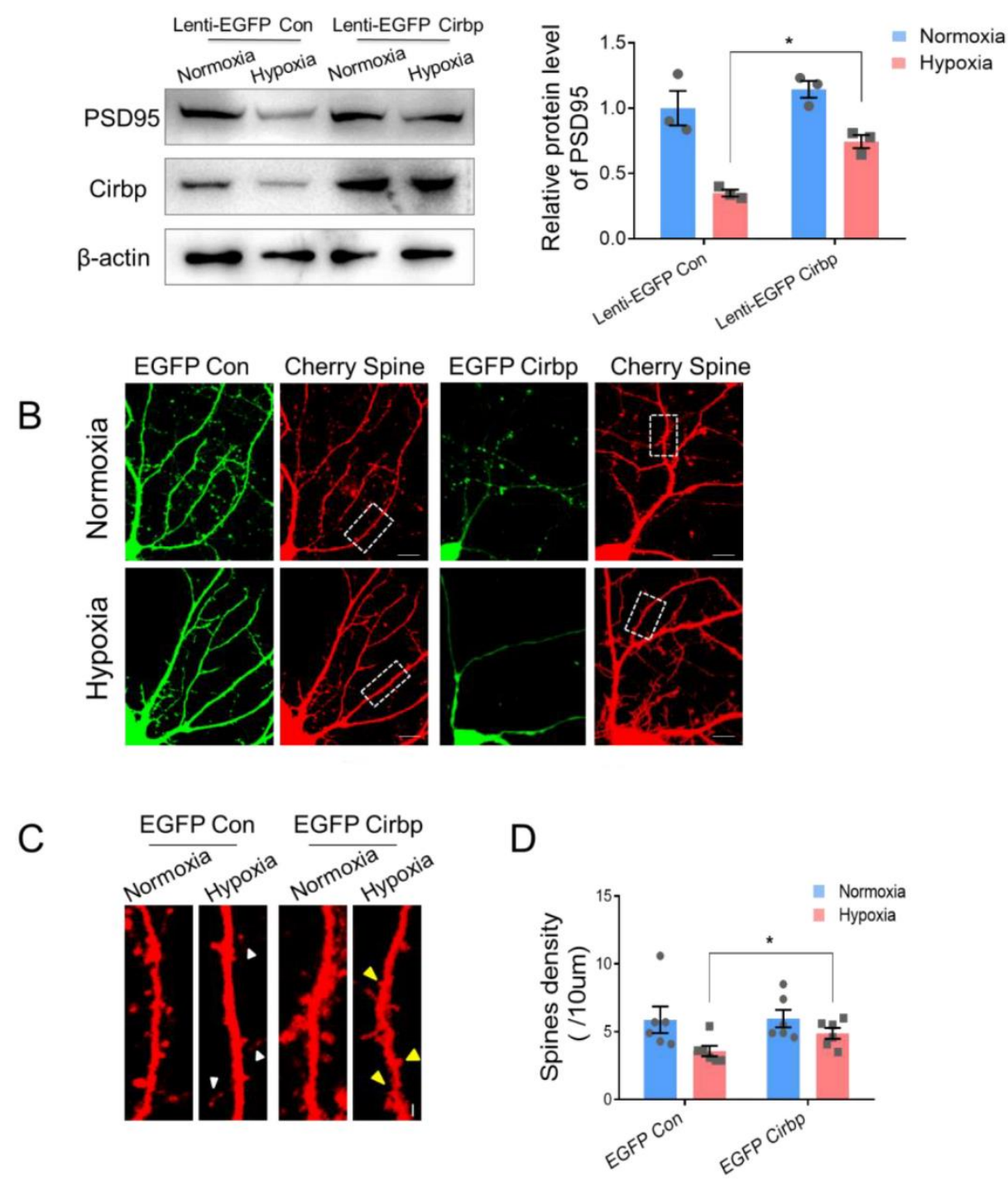

Figure 4 Effects of over-expression of Cirbp in primary hippocampal neurons exposed to hypoxia on PSD95 expression and the spine morphology.

(A) The protein levels of Cirbp and PSD95 in Lenti-EGFP con and Lenti-EGFP Cirbp infected primary hippocampal neurons ( $\mathrm{n}=3$ biological replicates, two-way ANOVA, $756 \pm$ SEM)

(B) Fluorescence images of EGFP Cirbp and mCherry-3FLAG lentivirus infected

758 primary hippocampal neurons under normoxia or hypoxia exposure, scale bar $=10 \mu \mathrm{m}$.

(C) The zoomed picture of dendritic spines, corresponds to the in the white frame of (B) via laser confocal microscopy (white triangle indicated filopodia and yellow triangle 
761 indicated the site of spine formation, scale bar $=5 \mu \mathrm{m}$ ).

762 (D) The statistical analysis of dendritic spine density (12 neurons / 3 mice per group,

763 two-way ANOVA, \pm SEM).

$764 * p<0.05$.

765 
A

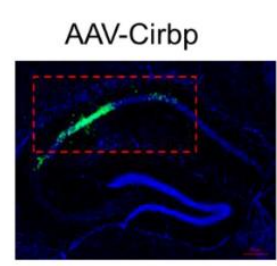

C

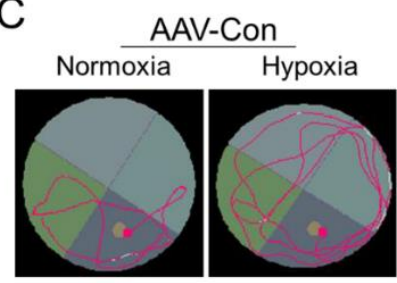

E

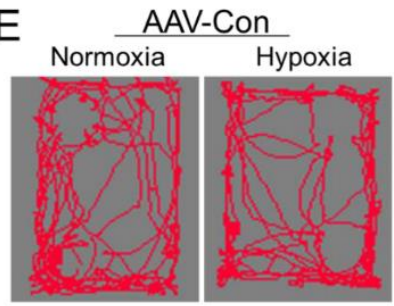

G

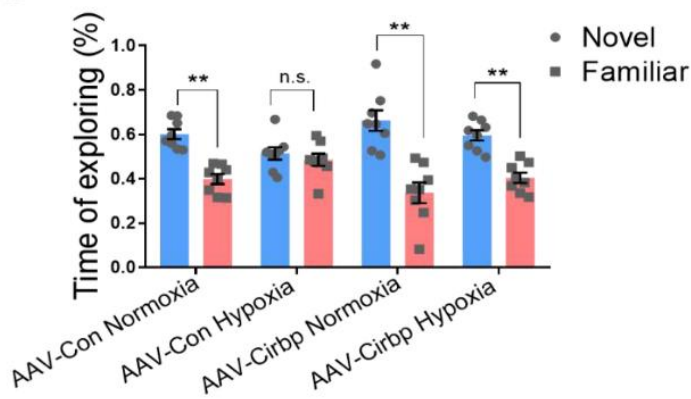

B Mouse Hippocampus
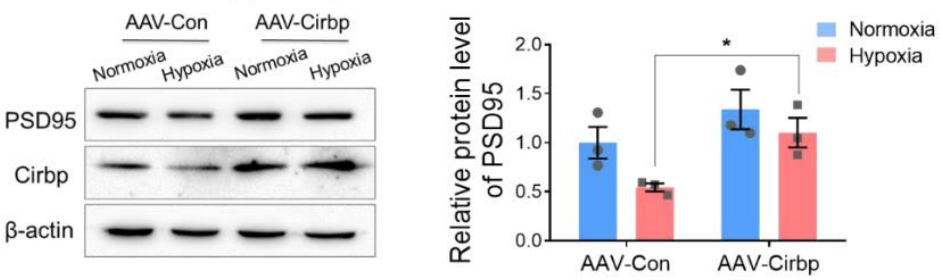

D

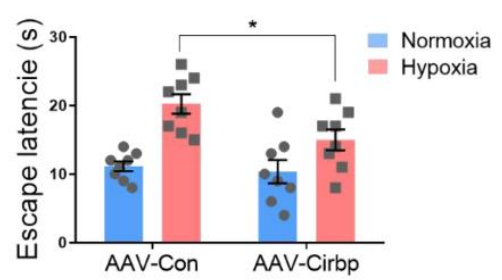

F

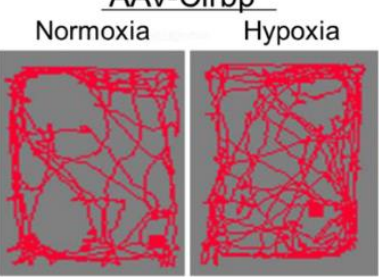

$\mathrm{H}$
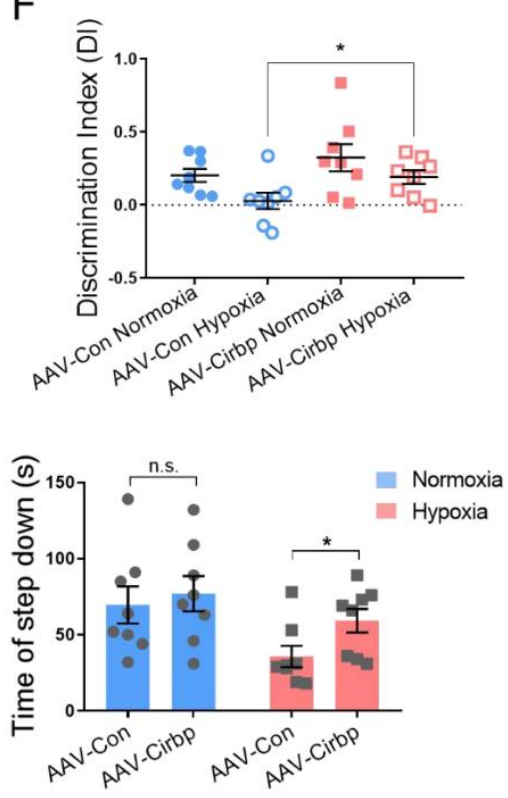

Figure 5 Ectopic expression of Cirbp alleviated memory dysfunction in hypobaric hypoxia exposed mice.

(A) Fluorescence image of mouse hippocampal CA1 region (red frame area) after stereotactic injection showing Cirbp expression (green) and Nucleus (blue), scale bar = $50 \mu \mathrm{m}$.

(B) Protein expression of PSD95 in mouse hippocampus following hypoxia exposure after over-expressing Cirbp ( $\mathrm{n}=3$ biological replicates, two-way ANOVA, \pm SEM).

(C-D) Representative tracking plots (C) and the escape latency (D) of mice under indicated treatment in MWM test (n=3, two-way ANOVA, \pm SEM). 
777 (E-G) Representative locomotion tracking plots (E), discriminate index (F) and 778 exploring time on new objects $(\mathrm{G})$ of mice under indicated treatment in NORT test $(\mathrm{n}=8$, 779 three-way ANOVA, \pm SEM)

$780(\mathrm{H})$ The latency time of step down in SIAT test ( $\mathrm{n}=8$, two-way ANOVA, \pm SEM)

781 n.s., no significant, $* p<0.05, * * p<0.01$.

782 
Figure 6

A

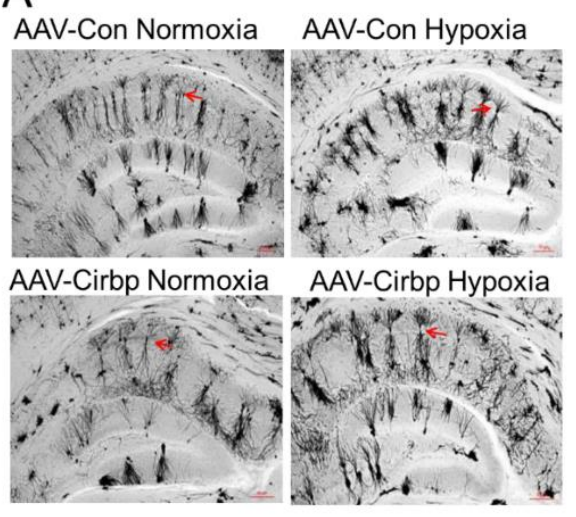

D

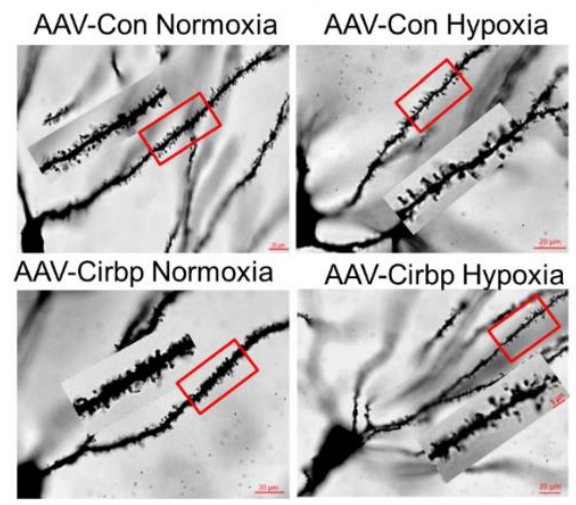

F
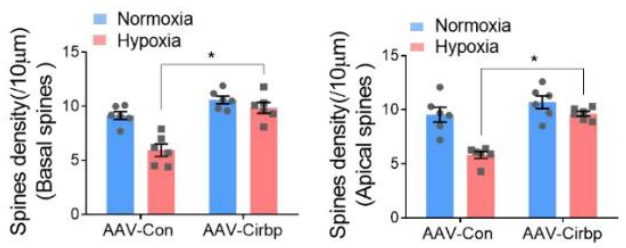

G
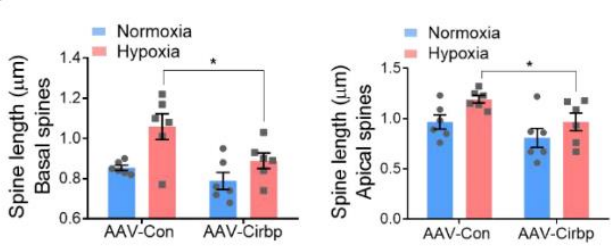

B

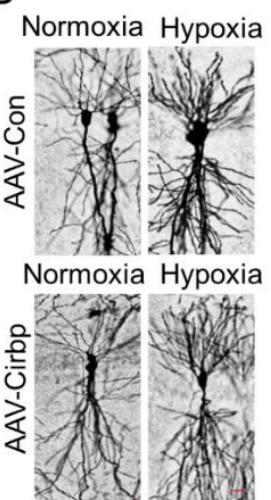

C

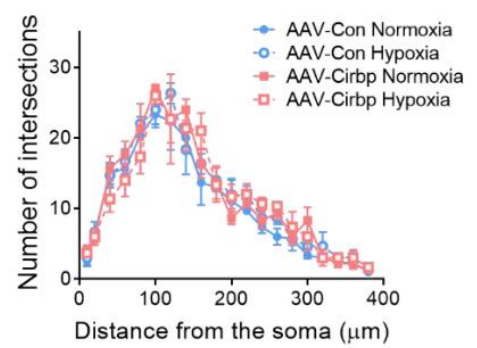

E Apical spines

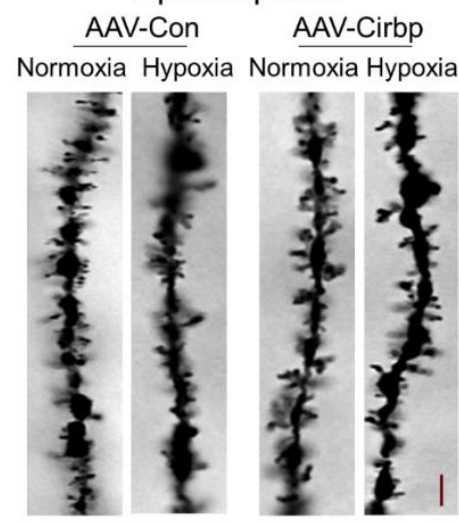

$\mathrm{H}$

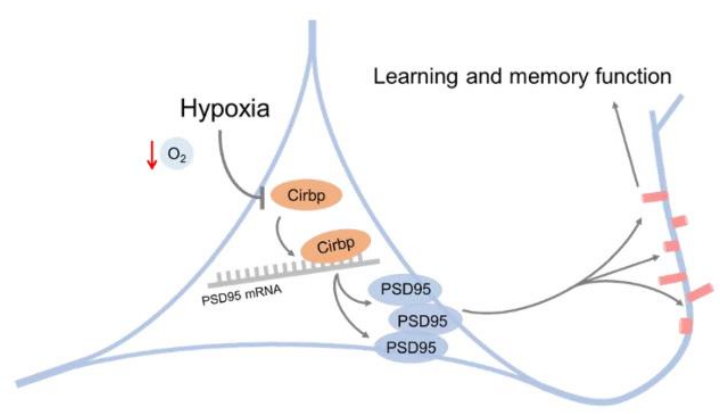

784

Figure 6 Cirbp alleviated hypoxia-induced dendritic spine abnormalities in mice

\section{hippocampus.}

(A-B) Golgi staining of mice hippocampus (A, scale bar $=50 \mu \mathrm{m})$ and of CA1 pyramidal neurons $(B$, scale bar $=20 \mu \mathrm{m})$.

(C) Number of intersections of pyramidal neuron reconstruction (12 neurons / 3 mice per group, two-way ANOVA, \pm SEM).

(D) Representative Golgi staining morphology of apical dendritic spines in CA1, scale 
792 bar $=20 \mu \mathrm{m}$ (red frame indicated target dendritic, scale bar=5 $\mu \mathrm{m})$.

793 (E) Representative morphological images of apical dendritic spines in CA1, scale bar $794=5 \mu \mathrm{m}$.

795 (F) Quantitative analysis of basal and apical spines density (12 neurons / 3 mice per 796 group, two-way ANOVA, \pm SEM).

797 (G) Quantitative analysis of basal and apical neck length (12 neurons / 3 mice per group, 798 two-way ANOVA, \pm SEM).

799 (H) A model for key role of Cirbp-PSD95 axis in hypoxia-induced hippocampal neuron 800 dendritic spine abnormality.

$801 * p<0.05$

802

803 
A

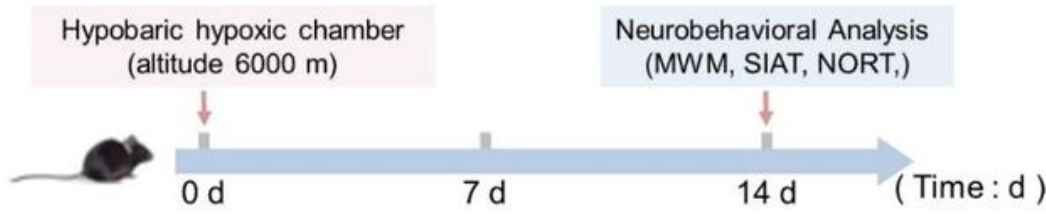

B

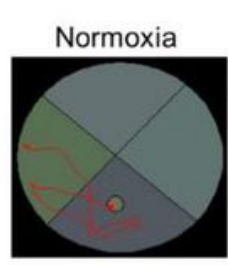

E
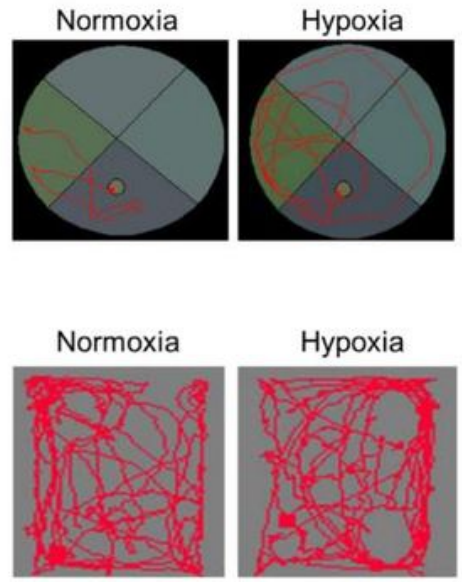

C

F

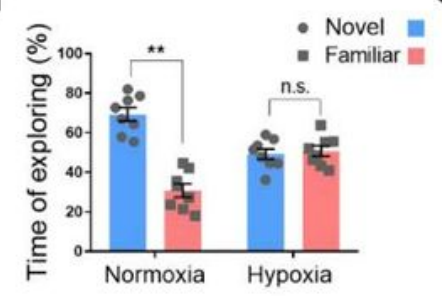

D

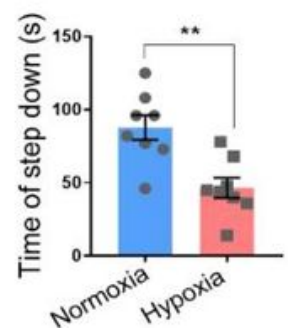

$G$

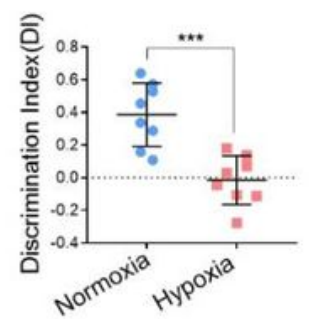

$\mathrm{H}$

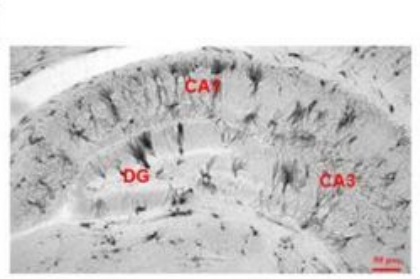

J

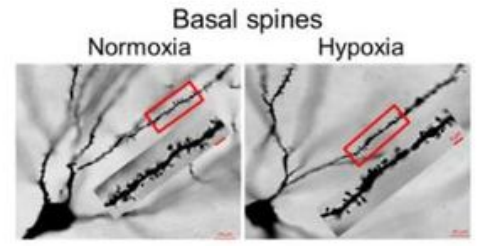

Normoxia
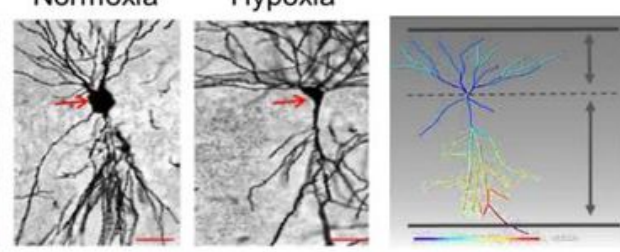

Apical spines

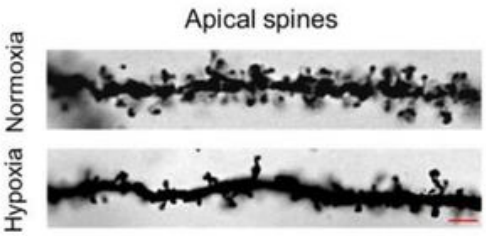

L

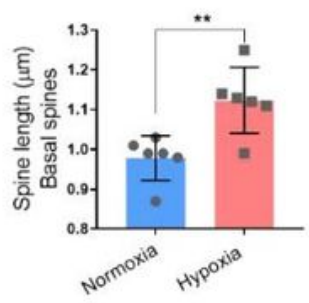

- Normoxia

- Hypoxia
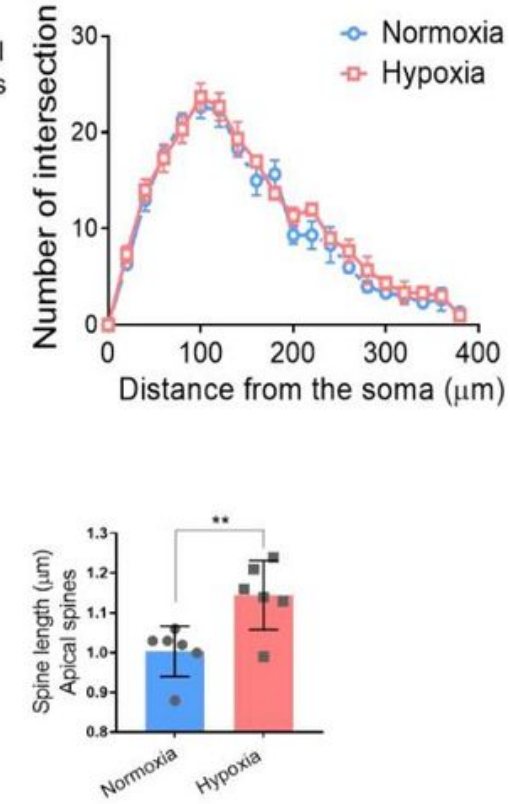

\section{Figure 1}

Hypobaric hypoxic exposure impaired memory ability and spine morphology. (A) The scheme of the experimental procedure. (B-C) Representative locomotion tracking plots (B) and the escape latency (C) of mice in MWM test $(n=8$, Student's t-test, $\pm S E M)$. (D) The latency time prior to descent from the platform in 
SIAT test ( $n=8$, Student's t-test, \pm SEM). (E-G) Representative locomotion tracking plots (E), exploring time on new objects ( $F)$ and the discriminate index $(G)$ in NORT test $(n=8$, Student's t-test, $\pm S E M)$. (H) Left: Representative golgi staining of mice hippocampus. DG, dentate gyrus, scale bar=50 $\mu \mathrm{m}$. Middle: Golgi staining of CA1 pyramidal neurons, scale bar $=20 \mu \mathrm{m}$. Right: Imaris reconstruction of CA1 pyramidal neurons. (I) Number of dendritic intersections of reconstructed pyramidal neurons by Sholl analysis (12 neurons/3 mice per group, Student's t-test, \pm SEM). (J) Left: Representative Golgi staining of basal dendritic spines in CA1, scale bar $=20 \mu \mathrm{m}$ (Red frame indicated target area, scale bar $=5 \mu \mathrm{m}$ ). Right: Representative morphology of apical dendritic spines in CA1, scale bar $=5 \mu \mathrm{m}$. (K) Quantification of basal and apical dendritic spines density and columns represent number of spines per $10 \mu \mathrm{m}$ (12 neurons / 3 mice per group, Student's t-test, $\pm S E M$ ). (L) Quantification of basal and apical neck length (12 neurons / 3 mice per group, Student's t-test, \pm SEM). ${ }^{\star} p<0.05,{ }^{\star \star} p<0.01,{ }^{\star \star \star} p<0.001$. 
A

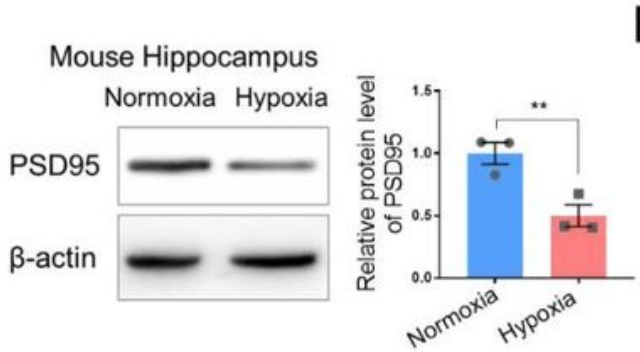

B

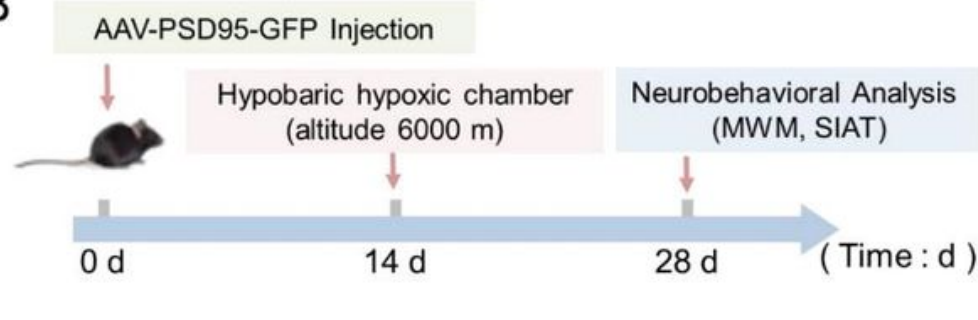

C

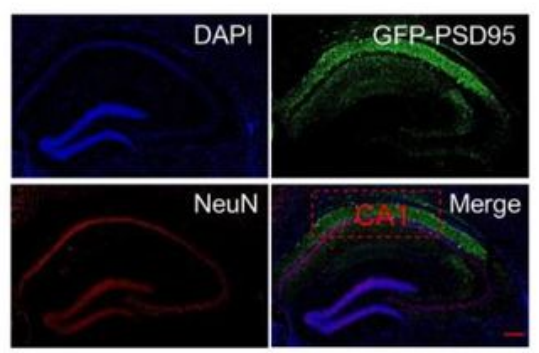

E

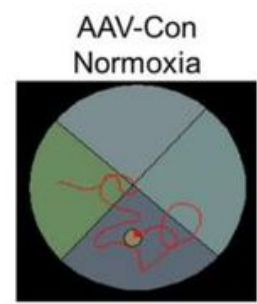

AAV-PSD95 Normoxia
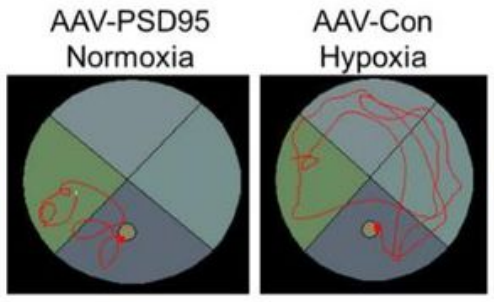

G
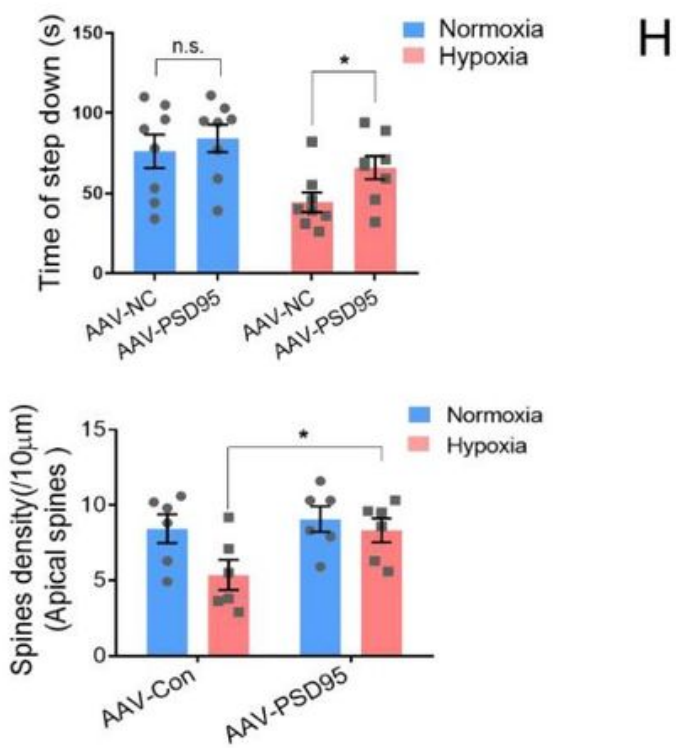

$\mathrm{H}$
AAV-PSD95 F Hypoxia
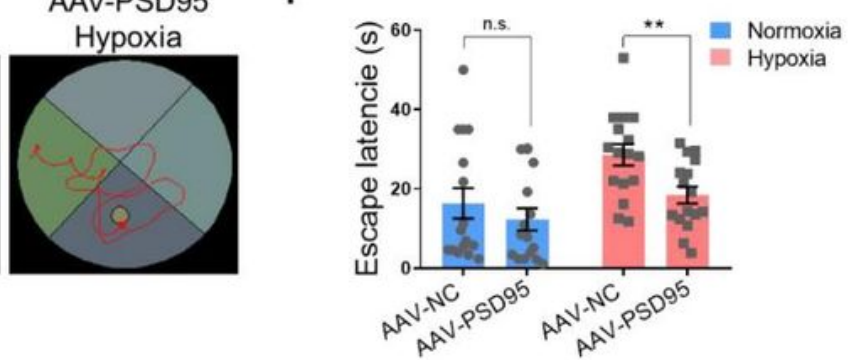

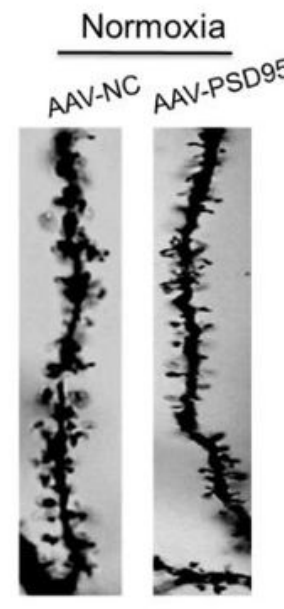

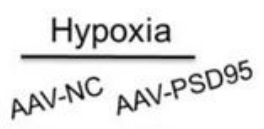
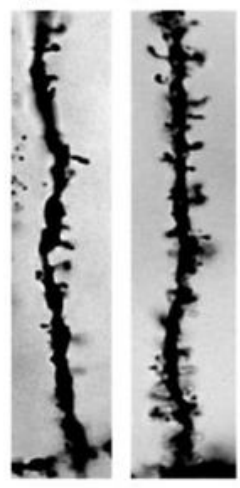

\section{Figure 2}

Hypobaric hypoxia down-regulated PSD95 protein level in mice hippocampus and ectopic expression of PSD95 restored memory function and the spine phenotype caused by hypoxic exposure. (A) Representative immunoblotting and the quantification analysis of PSD95 ( $n=3$ biological replicates, Student's t-test, \pm SEM) (B) Schematic representation of the experimental setup. (C) The fluorescence image of the hippocampus CA1 after stereotacitic injection showing the autofluorescence of AAV-PSD95 
(green) and DAPI (blue) and the immunofluorescence of NeuN (red), scale bar = $50 \mu \mathrm{m}$. (D)

Representative immunoblotting of PSD95. ( $n=3$ biological replicates). (E-F) Representative tracking plots $(E)$ and the escape latency $(F)$ of mice under indicated treatment in MWM test $(n=8$, two-way ANOVA, \pm SEM). (G) The latency time of mice under indicated treatment in SIAT test ( $n=8$, two-way ANOVA, \pm SEM). $(\mathrm{H}-\mathrm{I})$ Representative Golgi staining morphology $(\mathrm{H}$, scale bar=5 $\mu \mathrm{m})$ and quantitative analysis of density (I) of apical spines in hippocampus CA1 neurons of mice under indicated treatment (12 neurons / 3 mice per group, two-way ANOVA, \pm SEM) n.s., no significant, * $p<0.05$, ** $p<0.01$.

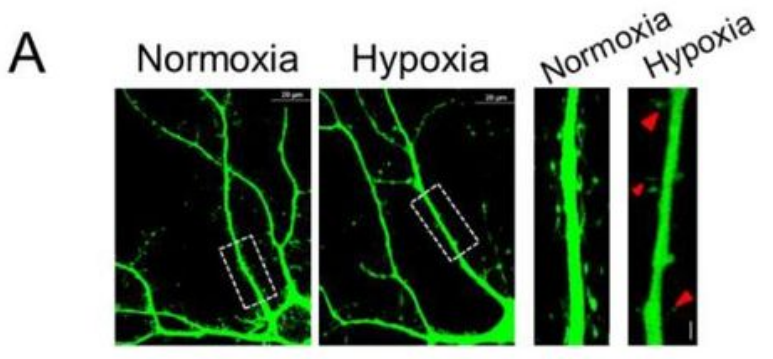

B

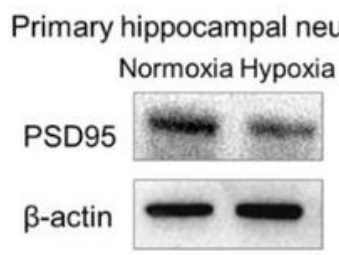

$\mathrm{E}$

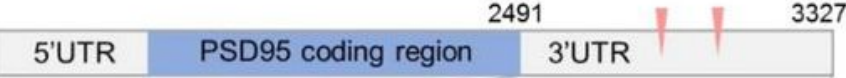

TTCCTGCCCTGGCTTGGCCTGGACTCACCCTGCCTCCACCACCTGGGCCCTTG GTCTGGACTGAATAGCCCAAGCCCTTCGTACCCTAGCCTTTCTTCCCACCCTTC TTATTTATTTCCTTTCTGACTGGATCGAGCCCATTGGAGGGAGGACACTCCTCTC TGCATGTATCCCTGCACCCCAGATCAGGGCCCCTAGACCCCAGGAACCTGGGG TCTGGGGGGGAGCTGGGCTCCTGGTTCCGAGCCCTTGCTCCTTAAGACTCCTA CCCCTGCCCGCCCCCATGCACACACAGACCCACTGGGGGCCTCTGCCCTCCC CATTTTCTCCCACACACATTCCAGAAGTCAGGGCCCCTCTCAAGGAGTACCCGC TGTAGGGATGCAGGGCCACAGGCTTCCGCGCTCTCCCGAGGCAGGGGCTGGG GTCACCCCTGCCCCATCATAACTCCCCACGCCGTTTGAGTTCTCCTTTATTTTCT CCATTCTITTCTTCTCAAAGGTGGTTITTGGGGGGAGAAGCAGGGGGGCTCTC CTGAGGGTCCCCCCGTCTTTCACACACCTCCCACCTTTITTCTTTGCCGGTTTG CATGAGTGGAAGGTCTAACTGTGGCTTITITITITTCTGGGATTITATTTCATTIT ATTTGATTTTATTITTITGGGAAAAGGGGAGGGATGGGTCTGGGGAGTGGGGAA TGTGGGAGGGGGGTGGGGGGGCAAAGGGTCAGGGGTTGGGTGTCCGGGAGC CAGGGGAGGACAGGAAATGCTGCCGCCTTCTGCAATTTATTTATTTTTTTCTTTT GAGAGAGTGAAAGGAAGAGACAGACACTTGAAAAAAAAAAAAAAA

G

Primary hippocampal neurons

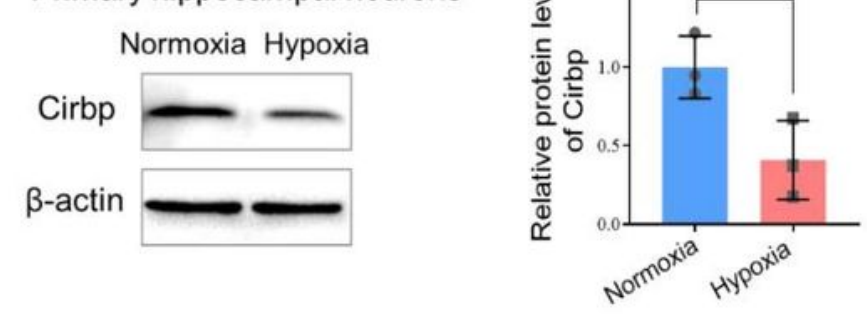

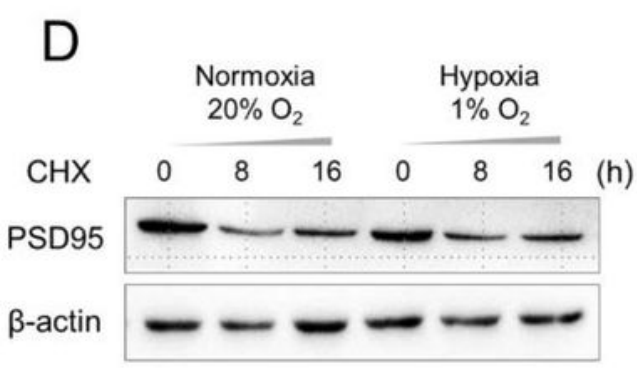

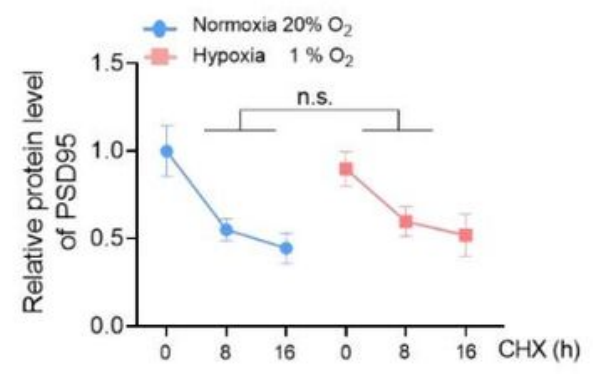

3'UTR-A

$2491-2769$

3'UTR-B

$2770-\cdots-3048$

3'UTR-C

$3049 \cdots 3327$

$\mathrm{F}$

| Cirbp conversive binding motif
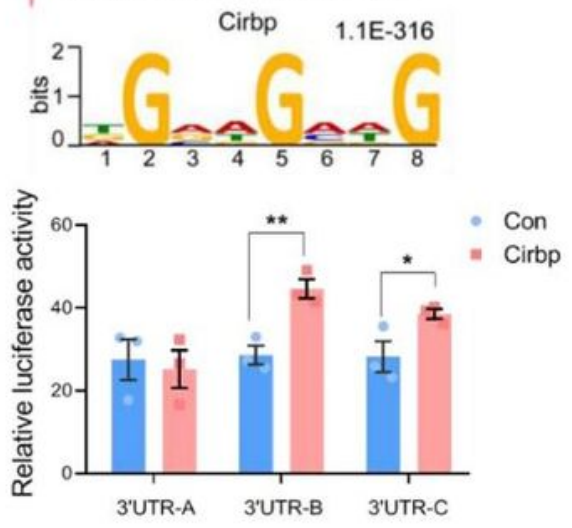

$\mathrm{H}$

Cirbp

Input $\lg G$ Cirbp

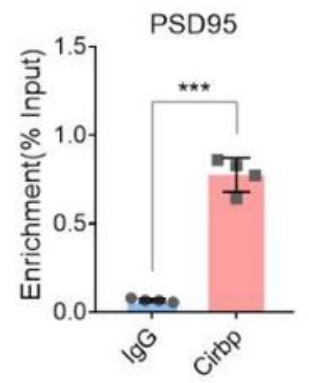




\section{Figure 3}

Hypoxic exposure resulted in aberrant dendritic spine morphology and PSD95 expression in vivo and Cirbp regulated PSD95 expression through 3'UTR binding. (A) Left: representative images of hippocampal neurons with hypoxia or normoxia exposure, scale bar $=10 \mu \mathrm{m}$. Middle: enlarged images of hippocampal neurons, red triangle indicated the elimination of spines or filopodia, scale bar $=5 \mu \mathrm{m}$. Right, the quantification of spines density (12 neurons / 3 mice per group, Student's t-test, \pm SEM). (B) Representative immunoblotting (left) and quantitative analysis (right) of PSD95 in primary hippocampal neurons under hypoxic exposure ( $n=3$ biological replicates, Student's $t$-test, $\pm S E M)$ (C) The relative mRNA levels of PSD95 in primary hippocampal neurons under hypoxic exposure $(n=3$ biological replicates, Student's t-test, \pm SEM). (D) PSD95 expression after CHX treatment based on western blot and quantitative analysis ( $n=3$ biological replicates, paired Student's t-test, $\pm S E M$ ). (E) RBP binding motif distributing in PSD95 3'UTR and construction of a series of luciferase reporter vectors containing PSD95 3'UTR fragments. (F) Luciferase activity assays to examine the functional RBP motif sites in the PSD95 3'UTR regulated by Cirbp ( $n=3$ biological replicates, Student's t-test, $\pm S E M)$. (G) Representative immunoblotting (left) and the quantitative analysis (right) of Cirbp in primary hippocampal neurons after hypoxia exposure ( $n=3$ biological replicates, Student's t-test, \pm SEM). (H) RNA immunoprecipitation (RIP) followed by RT-PCR in HT-22 cells ( $n=4$ biological replicates, Student's t-test, $\pm S E M)$. ${ }^{*} p<0.05,{ }^{\star \star} p<0.01$, $\star \star \star p<0.001$. 
A Primary hippocampal neurons
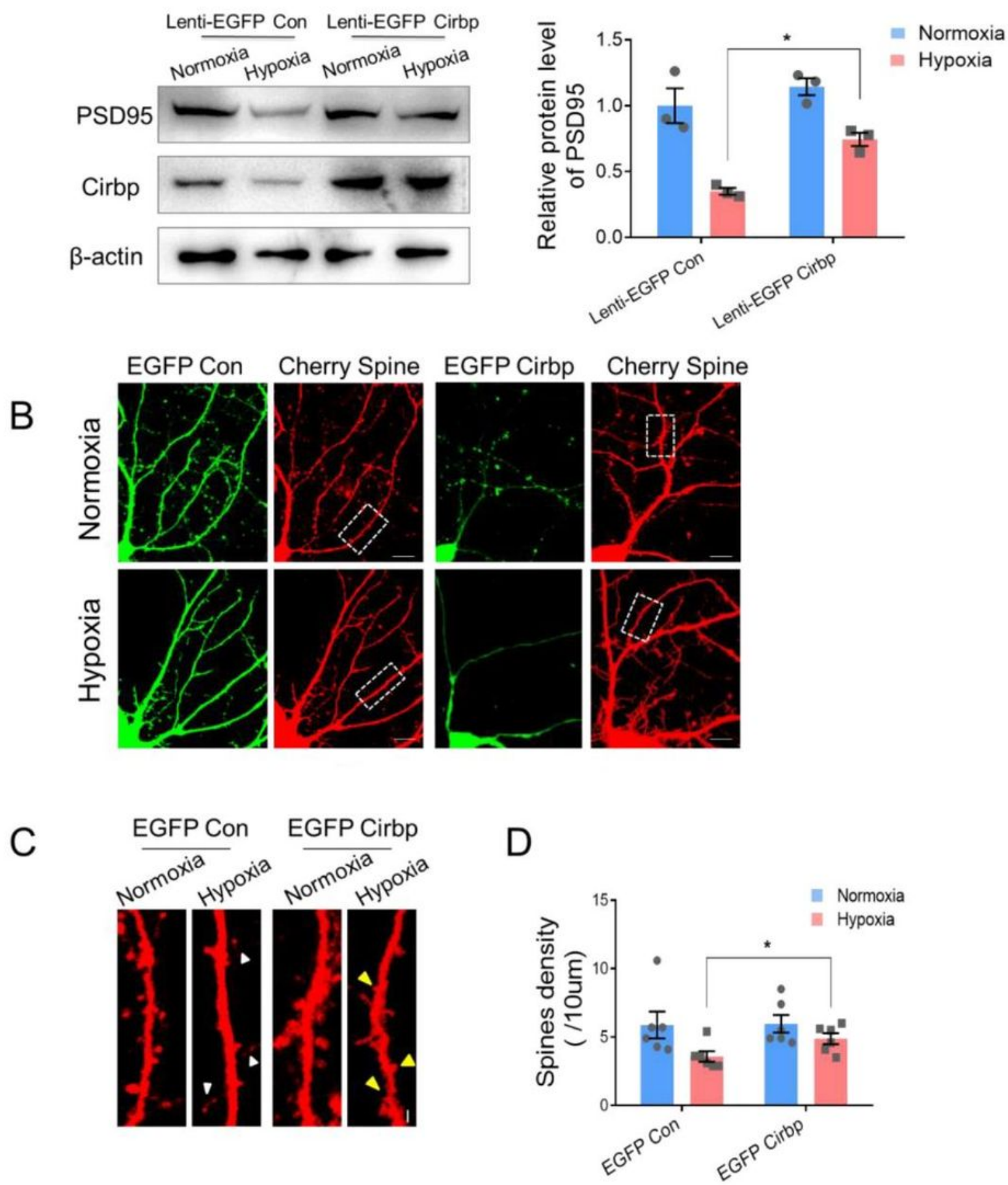

\section{Figure 4}

Effects of over-expression of Cirbp in primary hippocampal neurons exposed to hypoxia on PSD95 expression and the spine morphology. (A) The protein levels of Cirbp and PSD95 in Lenti-EGFP con and Lenti-EGFP Cirbp infected primary hippocampal neurons ( $n=3$ biological replicates, two-way ANOVA, \pm SEM) (B) Fluorescence images of EGFP Cirbp and mCherry-3FLAG lentivirus infected primary hippocampal neurons under normoxia or hypoxia exposure, scale bar $=10 \mu \mathrm{m}$. (C) The zoomed picture of 
dendritic spines, corresponds to the in the white frame of (B) via laser confocal microscopy (white triangle indicated filopodia and yellow triangle indicated the site of spine formation, scale bar $=5 \mu \mathrm{m}$ ). (D) The statistical analysis of dendritic spine density (12 neurons / 3 mice per group, two-way ANOVA, \pm SEM). * $p<0.05$.

A

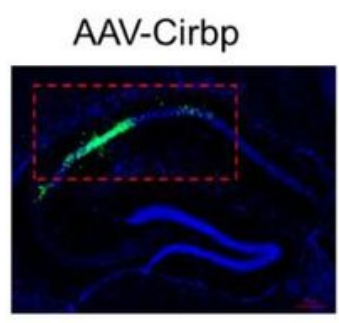

C

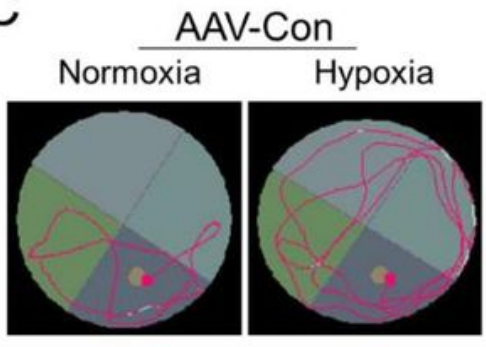

E
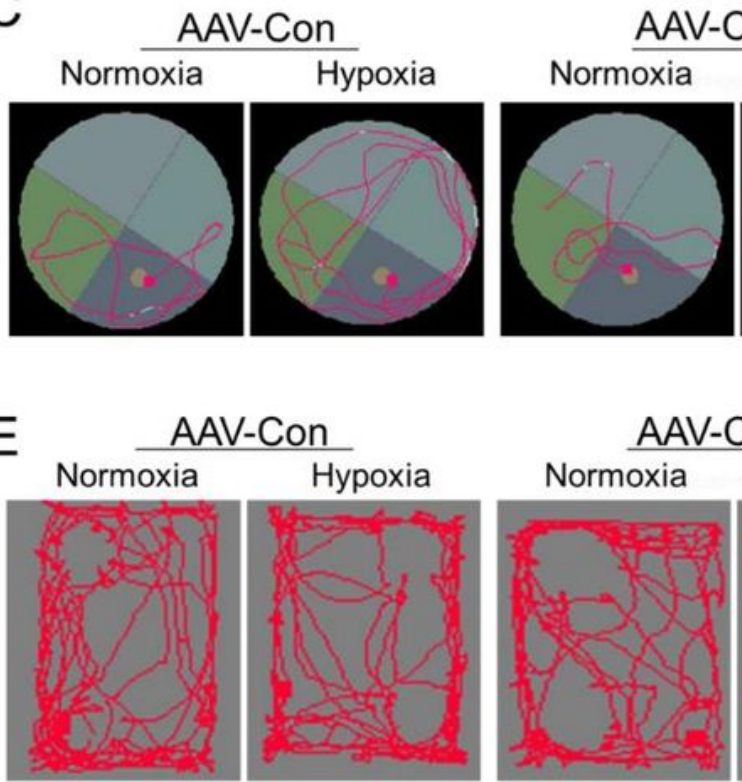

AAV-Cirbp

Hypoxia

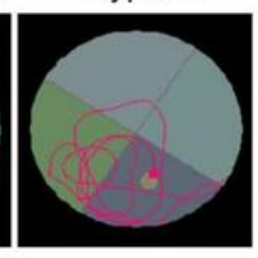

AAV-Cirbp
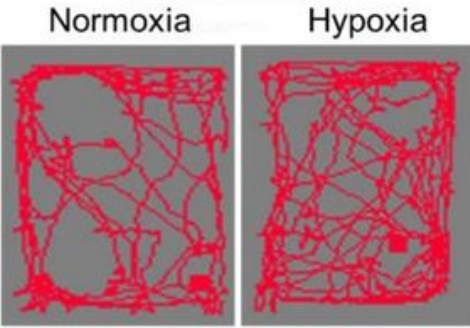

G

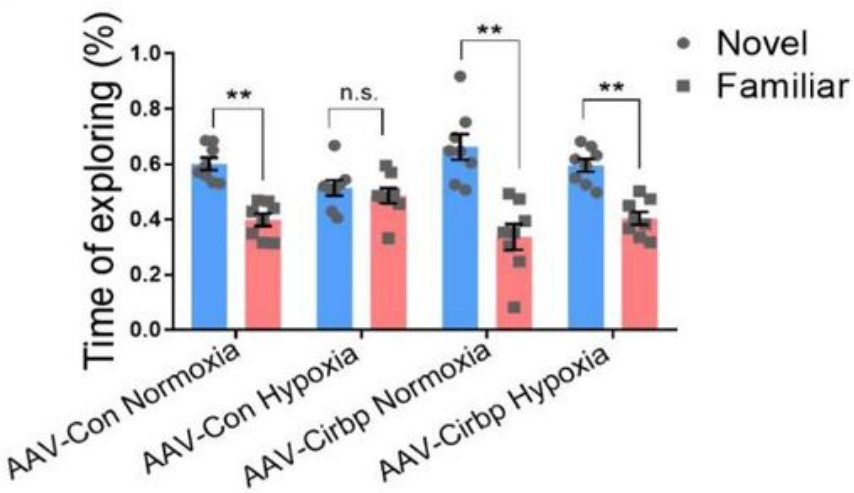

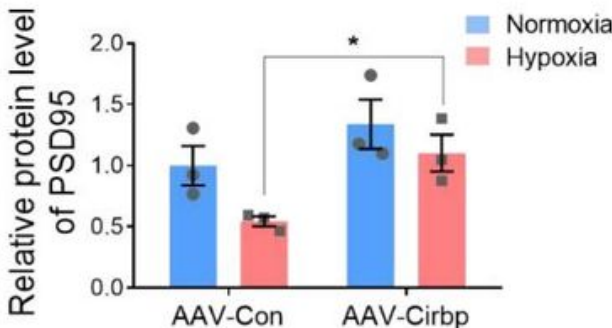

D

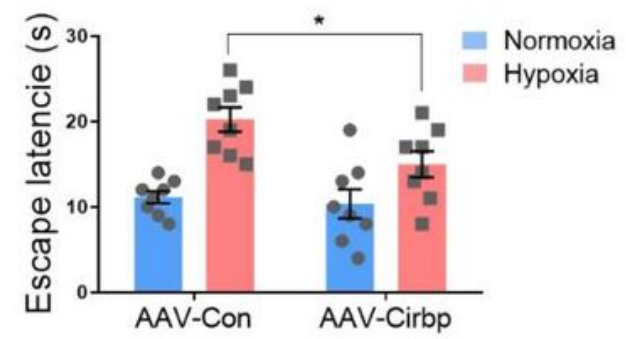

F

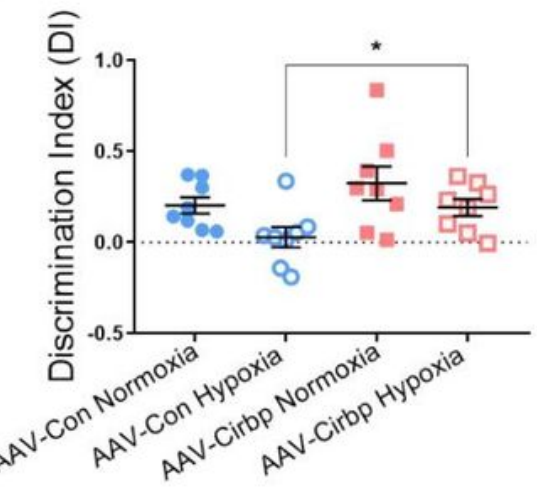

$\mathrm{H}$

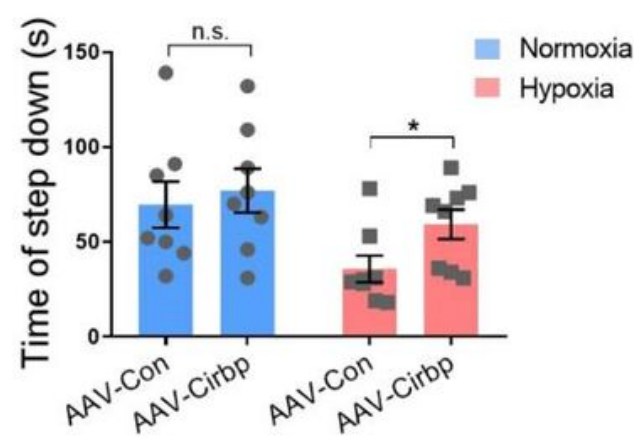

Figure 5 
Ectopic expression of Cirbp alleviated memory dysfunction in hypobaric hypoxia exposed mice. (A) Fluorescence image of mouse hippocampal CA1 region (red frame area) after stereotactic injection showing Cirbp expression (green) and Nucleus (blue), scale bar $=50 \mu \mathrm{m}$. (B) Protein expression of PSD95 in mouse hippocampus following hypoxia exposure after over-expressing Cirbp ( $\mathrm{n}=3$ biological replicates, two-way ANOVA, $\pm S E M$ ). (C-D) Representative tracking plots (C) and the escape latency (D) of mice under indicated treatment in MWM test ( $n=3$, two-way ANOVA, $\pm S E M)$. ( $E-G)$ Representative locomotion tracking plots $(E)$, discriminate index $(F)$ and exploring time on new objects $(G)$ of mice under indicated treatment in NORT test $(n=8$, three-way ANOVA, \pm SEM) $(H)$ The latency time of step down in SIAT test $(n=8$, two-way ANOVA, \pm SEM) n.s., no significant, $* p<0.05, * * p<0.01$. 
A

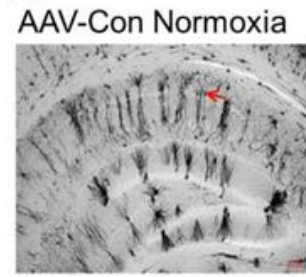

AAV-Con Hypoxia

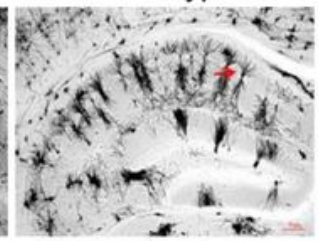

AAV-Cirbp Normoxia

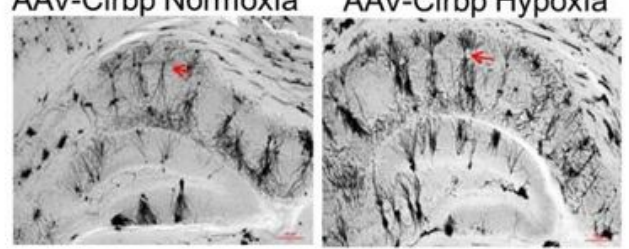

D

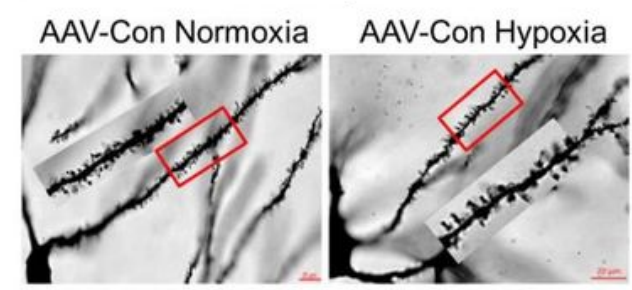

AAV-Cirbp Normoxia

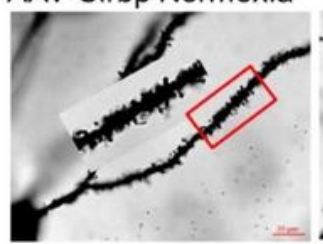

AAV-Cirbp Hypoxia

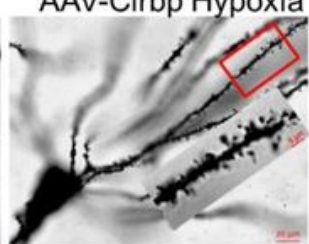

$\mathrm{F}$
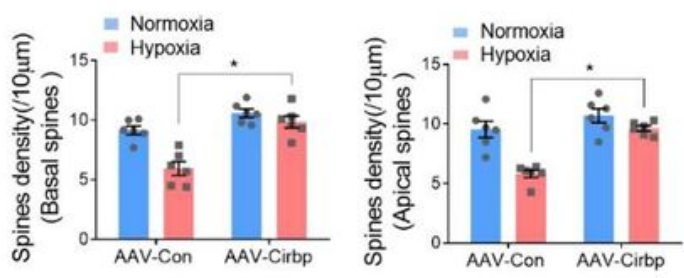

G

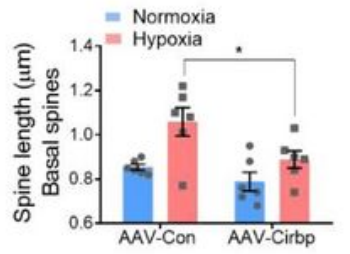

B
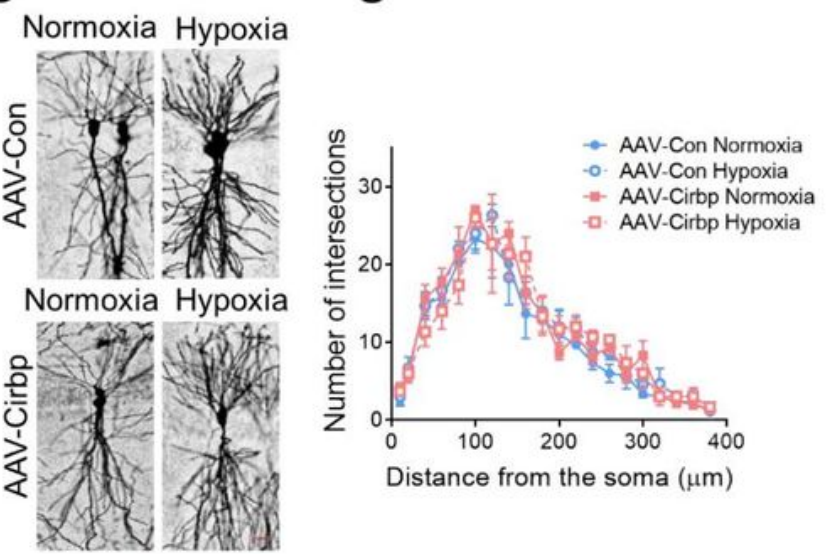

E

Apical spines

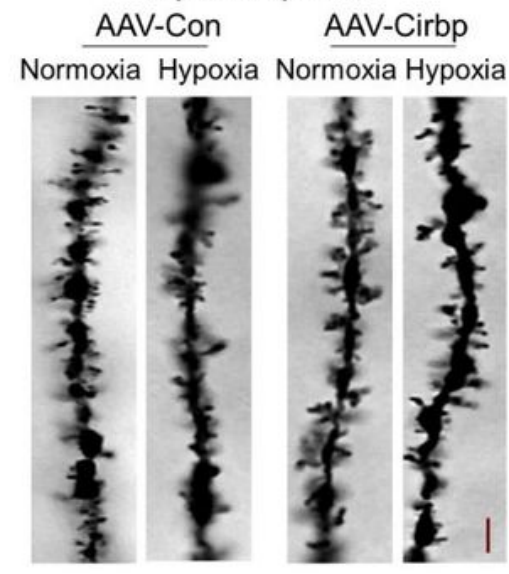

$\mathrm{H}$

\section{C}

ormoxia

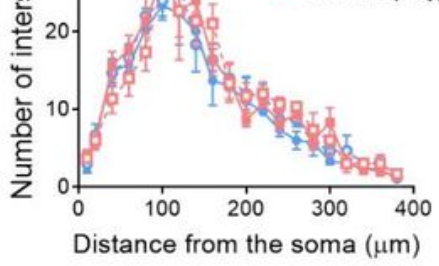

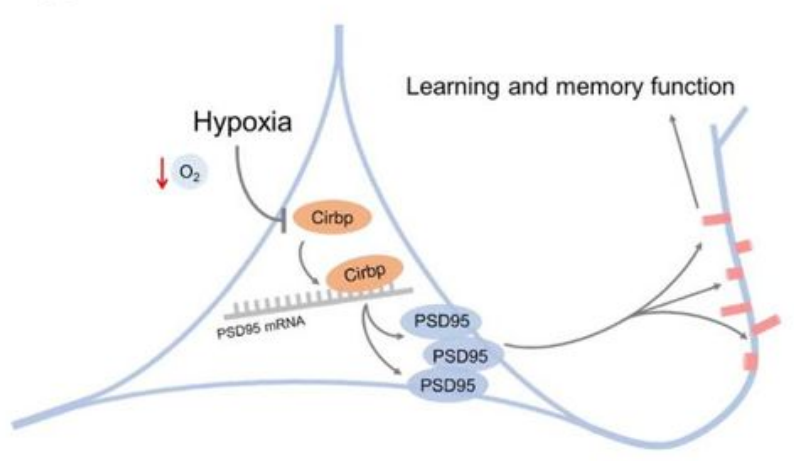

\section{Figure 6}

Cirbp alleviated hypoxia-induced dendritic spine abnormalities in mice hippocampus. (A-B) Golgi staining of mice hippocampus (A, scale bar=50 $\mu \mathrm{m}$ ) and of CA1 pyramidal neurons (B, scale bar=20 $\mu \mathrm{m})$. (C) Number of intersections of pyramidal neuron reconstruction (12 neurons / 3 mice per group, two-way ANOVA, \pm SEM). (D) Representative Golgi staining morphology of apical dendritic spines in CA1, scale bar $=20 \mu \mathrm{m}$ (red frame indicated target dendritic, scale bar $=5 \mu \mathrm{m}$ ). (E) Representative morphological images 
of apical dendritic spines in CA1, scale bar $=5 \mu \mathrm{m}$. (F) Quantitative analysis of basal and apical spines density (12 neurons / 3 mice per group, two-way ANOVA, \pm SEM). (G) Quantitative analysis of basal and apical neck length (12 neurons / 3 mice per group, two-way ANOVA, \pm SEM). (H) A model for key role of Cirbp-PSD95 axis in hypoxia-induced hippocampal neuron dendritic spine abnormality. ${ }^{*} p<0.05$.

\section{Supplementary Files}

This is a list of supplementary files associated with this preprint. Click to download.

- SupplementaryMaterials.pdf 